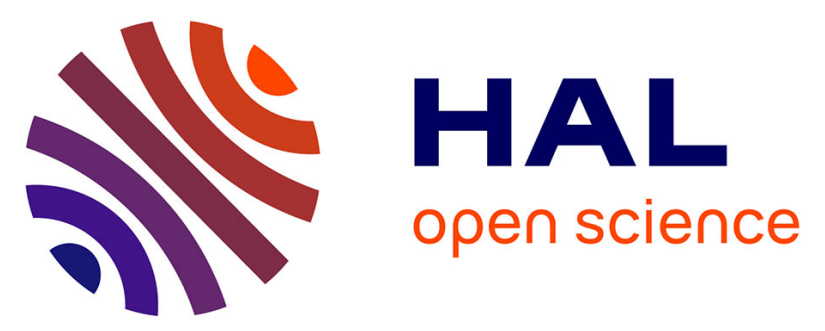

\title{
Sensitivity analysis of two-dimensional steady-state aquifer flow equations. Implications for groundwater flow model calibration and validation
}

Naomi Mazzilli, Vincent Guinot, H. Jourde

\section{- To cite this version:}

Naomi Mazzilli, Vincent Guinot, H. Jourde. Sensitivity analysis of two-dimensional steady-state aquifer flow equations. Implications for groundwater flow model calibration and validation. Advances in Water Resources, 2010, 33 (8), pp.905-922. 10.1016/j.advwatres.2010.04.014 . hal-00804654

\section{HAL Id: hal-00804654 \\ https://hal.science/hal-00804654}

Submitted on 26 Mar 2013

HAL is a multi-disciplinary open access archive for the deposit and dissemination of scientific research documents, whether they are published or not. The documents may come from teaching and research institutions in France or abroad, or from public or private research centers.
L'archive ouverte pluridisciplinaire HAL, est destinée au dépôt et à la diffusion de documents scientifiques de niveau recherche, publiés ou non, émanant des établissements d'enseignement et de recherche français ou étrangers, des laboratoires publics ou privés. 


\title{
Sensitivity analysis of two-dimensional steady-state aquifer flow equations. Implications for groundwater flow model calibration and validation
}

\author{
N. Mazzilli ${ }^{\mathrm{a}, *}$, V. Guinot ${ }^{\mathrm{a}}$, H. Jourde ${ }^{\mathrm{a}}$ \\ ${ }^{a}$ Hydrosciences Montpellier, UMR 5569 (CNRS, IRD, UM1, UM2), Université Montpellier 2, CC MSE, \\ 34095 Montpellier Cedex 5, France
}

\begin{abstract}
This paper presents the analytical properties of the sensitivity of the two-dimensional, steady-state groundwater flow equation to the flow parameters and to the boundary conditions, based on the perturbation approach. These analytical properties are used to provide guidelines for model design, model calibration and monitoring network design. The sensitivity patterns are shown to depend on the nature of both the perturbed parameter and the variable investigated. Indeed, the sensitivity of the hydraulic head to the hydraulic conductivity extends mainly in the flow direction, while the sensitivity to the recharge spreads radially. Besides, the sensitivity of the flow longitudinal velocity to the hydraulic conductivity propagates in both the longitudinal and transverse directions, whereas the sensitivity of the flow transverse velocity propagates in the diagonal directions to the flow. The analytical results are confirmed by application examples on idealized and realworld simulations. These analytical findings allow some general rules to be established for model design, model calibration and monitoring network design. In particular, the optimal location of measurement points depends on the nature of the variable of interest. Measurement network design thus proves to be problem-dependent. Moreover, adequate monitoring well network design may allow to discriminate between the possible sources of error.
\end{abstract}

Keywords: perturbation approach, model sensitivity, model calibration, well network design, groundwater flow modelling

\section{Introduction}

Sensitivity analysis is the study of a system response to disturbances [1], and is now recognized as an integral part of the modelling process [2]. In the field of water resources, its range of application includes scenario analysis [3], optimization [4, 5], identification of the relevant parameters to model calibration [6] and experimental network design $[7,8]$.

In this paper, sensitivity analysis is used to derive some general rules for two-dimensional groundwater flow model calibration and monitoring network design. Since the first step in the calibration process is a steady-state simulation, the analysis is carried out for steadystate, two-dimensional flow simulations. The analytical properties of the sensitivity of the steady-state two-dimensional groundwater flow equation to the flow parameters and to the

\footnotetext{
* Corresponding author

Email address: mazzilli@msem.univ-montp2.fr (N. Mazzilli)
} 
boundary conditions are investigated, based on the perturbation approach. The main issues addressed are: (i) do flow parameters, model geometry and boundary conditions influence model response in the same way, (ii) do confined and unconfined aquifers behave in the same way with respect to the sensitivity propagation, (iii) when a two-dimensional model is shown to provide wrong simulation results, where should additional measurements be carried out in priority, and (iv) can general rules be defined for the optimal location of measurement points? These issues have been previously addressed for the steady state, two-dimensional shallow water flow equations [9]. The present work follows the methodology described in this former study.

In most studies available from the litterature, the effects of a perturbation on the response of a groundwater flow system have been investigated with the concern of understanding the effects of the heterogeneity of the governing hydraulic parameters distribution on hydraulic tests $[10,11,1,12,13,14,15$, e.g.]. A review of papers on the actual meaning of the transmissivity estimates derived from drawdown data collected in pumping wells in heterogeneous aquifers can be found in [13]. Analytical solutions for transient, pumping-induced drawdown sensitivity in non-uniform aquifers were derived by means of a first-order sensitivity formalism for simplified heterogeneity geometries by various authors $[10,11,1,16,17,14]$ based on the Theis equation. McElwee [1] investigated the difference in the drawdown sensitivity behaviour with respect to the nature of the perturbed parameter (transmissivity or storage), based on the Theis equation. Changes in the storage were shown to induce a perturbation in the simulated drawdown over a larger area than changes in the transmissivity. Sykes et al. [18] developed the adjoint sensitivity equations for a two-dimensional steady state flow in a confined aquifer. Sensitivities of local hydraulic head and Darcy velocities (performance measure) with respect to elemental changes in the flow parameters and boundary conditions were computed for an aquifer with heterogeneous conductivity distribution. Butler and Liu [17] focused on the influence of an heterogeneity on the observed drawdown and rate of change in drawdown. Delay et al. [19] developed an approach to the interpretation of interference pumping tests in fractal dual media. Analytical sensitivity calculations indicated that dual media have a sequential response in time to the pumping stress. On the basis of numerical simulations, Jiao and Zheng [20] concluded that the information on transmissivity is transferred mainly from upstream to downstream, while the information on storativity is transferred equally upstream and downstream. Conclusions were drawn as to pumping-tests design. The validity of these results is restricted to one-dimensional flow and specific boundary conditions. The spatial structure of the sensitivity of the hydraulic head with respect to the permeability field was investigated by means of a direct differentiation method by Khan et al. [21]. A method for high-sensitivity zones spotting was proposed, based on the observed sensitivity patterns. A systematic pattern to the sign of the sensitivities was observed, as the sign of a sensitivity coefficient was noted to be a function of the relative location of the perturbation within the flow field with respect to the calibration point. Oliver [12] used a perturbation approach to evaluate the effects of two-dimensional areal variations in the aquifers transmissivity and storage on observation well drawdown, based on the Fréchet derivatives and kernels. This work suggested that the area influencing observation well drawdowns were bounded by an ellipse enclosing the pumping and the observation wells. A detailed analysis of the spatial distribution of the hydraulic head sensitivity with respect to transmissivity and storage perturbations was performed for classical pumping test configurations $[22,23]$. Sensitivity coefficients were derived by means of the adjoint-state method. Sensitivity distributions of hydraulic tests with spatially separated stimulation and observation locations were shown to be characterized by a division of the domain of interest into two regions of opposite sensitivities. Based on the Theis approximation, Knight and Kluitenberg [24] derived explicit expressions of the Fréchet kernels and the spatial sensitivity 
functions for variations in storativity and transmissivity during both pumping and slug test. For not-colocated pumped and observation wells, both wells were shown to have the same importance with respect to spatial sensitivity, which confirmed previous results obtained by Leven [22]. The sensitivity of a groundwater flow system to the recharge boundary condition prescribed at the top boundary of a fully saturated groundwater flow model was investigated by Jyrkama and Sykes [25] by means of the adjoint method.

In most of the abovementioned studies, the sensitivity equation was solved using numerical methods. The analytical approach followed in this paper provides theoretical insights into the general behaviour of the two-dimensional, steady-state aquifer flow equations. The direct approach is used for the sensitivity computation. The direct sensitivity analysis is well suited to the computation of the sensitivity of several variables with respect to a given parameter. However, the computation of the sensitivity of a single variable with respect to multiple input parameters is best handled by an adjoint sensitivity analysis. The adjoint equation models similar physical processes as the direct equation, with a reversed flow of information. This means the direct and adjoint steady state sensitivity equations are the same, except that their velocity fields have opposite signs [26, 27]. The analytical properties of the sensitivity derived in the present paper thus apply to both the direct and adjoint frameworks.

The present work is structured in the following way. Section 2 presents the derivation of the two-dimensional steady-state groundwater sensitivity equations, based on the perturbation method. The solutions of the sensitivity equations and their analytical properties are investigated next. Sections 3 and 4 deal with perturbations in the flow parameters for confined and unconfined aquifers respectively. Section 5 handles the case of a perturbation in the boundary condition. Computational examples are presented in Section 6. Section 7 is devoted to conclusions.

\section{Sensitivity equations for 2-D aquifer flow}

\subsection{The groundwater flow equations}

Under Dupuit's hypothesis of negligible vertical flow, the steady state hydraulic head field satisfies the following equations [28]

$$
\begin{aligned}
-\nabla[K e \nabla H] & =R & & (\text { confined flow }) \\
-\nabla[K(H-z) \nabla H] & =R & & \text { (unconfined flow })
\end{aligned}
$$

where $H(\mathrm{~m})$ is the hydraulic head, $K(\mathrm{~m} / \mathrm{s})$ is the hydraulic conductivity (assumed independent from $H), z(\mathrm{~m})$ is the elevation of the aquifer bedrock, $R(\mathrm{~m} / \mathrm{s})$ is the recharge rate and $e(\mathrm{~m})$ is the aquifer thickness (independent from $H$ in the confined case).

\subsection{Sensitivity equations for confined aquifer flow}

The sensivity equations are derived by carrying out a perturbation analysis of the steady state groundwater flow equation. The reader interested in a more thorough discussion on sensitivity calculation can refer to $[29,30,31$, e.g.]. Let $\phi$ be the parameter with respect to which the sensitivity analysis is carried out. Assume that $\phi$ is modified by a small quantity $\phi_{o} \varepsilon(x, y)$ over a region $\Omega$, where $\phi_{o}$ is an infinitesimal constant and $\varepsilon(x, y)$ is the support function of the perturbation. Since $\phi$ may be any of the parameters of the flow equation, we denote the parameters of the perturbed equation $e+e^{\prime}, K+K^{\prime}$ and $R+R^{\prime}$ where $e^{\prime}$, $K^{\prime}$ and $R^{\prime}$ are the perturbations in the initial parameters $e, K$ and $R$ respectively. The solution of the perturbed diffusivity equation is the hydraulic head $H+H^{\prime}$. The perturbed flow equation thus becomes

$$
-\nabla\left[\left(K+K^{\prime}\right)\left(e+e^{\prime}\right) \nabla\left(H+H^{\prime}\right)\right]=R+R^{\prime}
$$


Subtracting equation (1a) from equation (2) and eliminating the second-order terms leads to

$$
\left.-\nabla\left[\left(K e^{\prime}+K^{\prime} e\right) \nabla H+K e \nabla H^{\prime}\right)\right]=R^{\prime}
$$

Dividing equation (3) by $\phi_{o}$ and defining the sensitivity of the hydraulic head to the parameter $\phi$ as $\eta_{\phi} \equiv \lim _{\phi_{o} \rightarrow 0} \frac{H^{\prime}}{\phi_{o}}$ leads to

$$
\left.-\nabla\left[\left(K \varepsilon_{e}+e \varepsilon_{K}\right) \nabla H+K e \nabla \eta_{\phi}\right)\right]=\varepsilon_{R}
$$

where

$$
\begin{aligned}
\varepsilon_{K} & =\frac{\partial K}{\partial \phi_{o}} \varepsilon(x, y) \\
\varepsilon_{e} & =\frac{\partial e}{\partial \phi_{o}} \varepsilon(x, y) \\
\varepsilon_{R} & =\frac{\partial R}{\partial \phi_{o}} \varepsilon(x, y)
\end{aligned}
$$

Note that

$$
\varepsilon_{\phi}= \begin{cases}\varepsilon(x, y) & \text { if } \phi \text { is the perturbed parameter } \\ 0 & \text { otherwise }\end{cases}
$$

The sensitivity equations may be rewritten in a more general manner

$$
-\nabla\left(K e \nabla \eta_{\phi}\right)=\rho
$$

with

$$
\rho= \begin{cases}\nabla(e \varepsilon \nabla H) & \text { if } \phi=K \\ \nabla(K \varepsilon \nabla H) & \text { if } \phi=e \\ \varepsilon & \text { if } \phi=R\end{cases}
$$

Equation (7) is a transport equation with a source term and diffusive effects. The source term is zero outside the perturbed area. Note that $H$ does not appear in equation (7) outside the perturbed area for the parameters $K$ and $e . H$ is not involved either in equation (7) inside or outside the perturbed area for the parameter $R$. Diffusivity is induced by $\nabla\left(K e \nabla \eta_{\phi}\right)$. Diffusive effects mean that a perturbation influences the value of $H$ over the whole domain.

The sensitivity of the velocity components to the parameter $\phi$ can be derived from the sensitivity of the hydraulic head to the same parameter using Darcy's law. The expression of the Darcy's velocity is identical for confined and unconfined aquifers

$$
v=-K \nabla H
$$

where $v$ is Darcy's velocity. Differentiating equation (9) with respect to the parameter $\phi$ gives

$$
\frac{\partial v}{\partial \phi}=-K \frac{\partial}{\partial \phi}(\nabla H)-\nabla H \frac{\partial K}{\partial \phi}
$$

Remember that

$$
\frac{\partial K}{\partial \phi}=\lim _{\phi_{o} \rightarrow 0} \frac{K^{\prime}}{\phi_{o}}
$$

If $\phi=K$ then $\phi_{o}=k_{o}$ and $K^{\prime}=k_{o} \varepsilon$, which leads to

$$
\frac{\partial K}{\partial \phi}=\varepsilon
$$


Denoting by $\omega_{\phi}$ the sensitivity of the flow velocity to the value of the parameter $\phi$ leads to

$$
\omega_{\phi}= \begin{cases}-K \nabla \eta_{\phi} & \text { if } \phi \neq K \\ -K \nabla \eta_{\phi}-\varepsilon \nabla H & \text { if } \phi=K\end{cases}
$$

Outside the perturbed area, $\varepsilon=0$ regardless of the parameter considered. Equation (13) thus simplifies into

$$
\omega_{\phi}=-K \nabla \eta_{\phi}
$$

\subsection{Sensitivity equations for unconfined aquifer flow}

Applying the perturbation approach used in Section 2.2 to equation (1b) for an unconfined aquifer leads to

$$
\begin{aligned}
& -\nabla\left[K \eta_{\phi} \nabla H+K(H-z) \nabla \eta_{\phi}\right]= \\
& \quad \nabla\left[\left(-K \varepsilon_{z}+(H-z) \varepsilon_{K}\right) \nabla H\right]+\varepsilon_{R}
\end{aligned}
$$

Equation (15a) may be rewritten as

$$
\nabla\left(v \eta_{\phi}\right)-\nabla\left[K(H-z) \nabla \eta_{\phi}\right]=\rho
$$

with

$$
\rho= \begin{cases}\nabla[(H-z) \varepsilon \nabla H] & \text { if } \phi=K \\ -\nabla(K \varepsilon \nabla H) & \text { if } \phi=z \\ \varepsilon & \text { if } \phi=R\end{cases}
$$

Equation (16) is a transport equation with a source term and diffusive effects. Remember that second-order terms have been neglected. The main difference with the confined case lies in the advective component $\nabla\left(v \eta_{\phi}\right)$, which means that the sensitivity is advected at the Darcy velocity. Assuming that the aquifer bedrock elevation is constant, equation (16) can be simplified into

$$
-\nabla\left[K \nabla\left((H-z) \eta_{\phi}\right)\right]=\rho
$$

When the perturbed parameter is the bedrock elevation or the recharge, the variable $(H-z) \eta_{\phi}$ obeys the same equation under unconfined conditions as does $\eta_{\phi}$ under confined conditions. In this case, sensitivity patterns under unconfined conditions are identical to those obtained for confined conditions, but distorted by a factor $1 /(H-z)$.

\section{Sensitivity properties for confined aquifers}

The theoretical developments are carried out for a homogeneous aquifer with a uniform, parallel flow directed in the negative $y$-direction (Figure 1). Anisotropic problems can be recast in the form of isotropic problems using a coordinate change. Whatever the parameter investigated, the support function of the perturbation $\varepsilon(x, y)$ is taken equal to zero everywhere except over a square zone $\Omega$ of size $2 L \times 2 L$ centred around $(0,0)$, where it varies linearly from 0 at the edges of the zone to 1 at its centre (see Figure 1). Note that any continuous support function could be decomposed with first-order accuracy into a sum of elementary support function such as $\varepsilon$. Such an approach is customary in e.g. finite element modelling. 

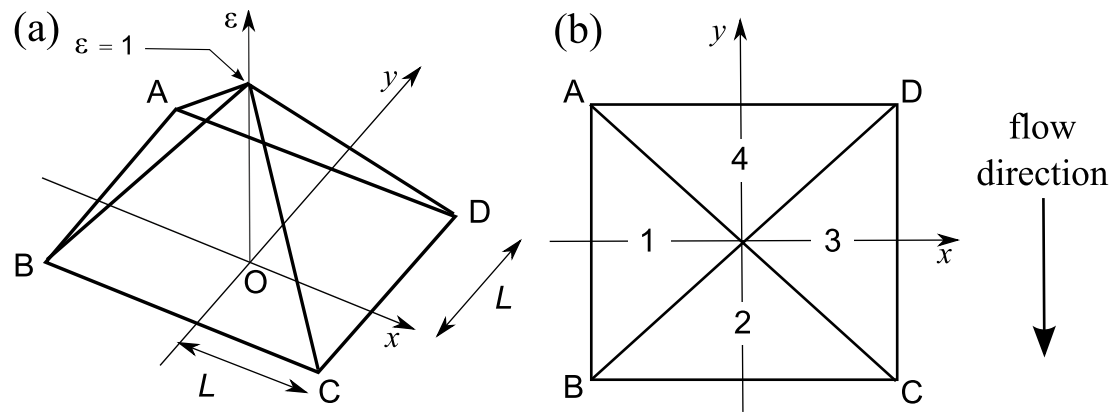

Figure 1: Definition sketch for $\varepsilon$ : (a) Perspective view, (b) Plan view

\subsection{Influence of a perturbation in the hydraulic conductivity for parallel flow}

Consider the case of a perturbation in the hydraulic conductivity. The source term in equation (7) is non-zero over the pertubed area only. At a distance $r$ from the perturbation such that $r>L$ (far field), the integral of the source term over the perturbed area can be approximated by a doublet of sources made of a sink with intensity $-e L \partial H / \partial y$ (point source 1 ) located at $(0,2 L / 3)$ and a source with intensity $+e L \partial H / \partial y \quad$ (point source located 2) at $(0,-2 L / 3)$ (see demonstration in Appendix A.1 and see source configuration in Figure 2 ). The field generated by this equivalent source configuration is known from the theory of potential flow [32] to be

$$
\eta_{K}=\frac{L}{2 \pi K} \frac{\partial H}{\partial y} \ln \left(\frac{r_{1}}{r_{2}}\right)
$$

where $r_{1}$ and $r_{2}$ are the distances to the equivalent point sources 1 and 2 respectively. The sign of $\eta_{K}$ is negative upstream of the perturbed area, and positive downstream. This result is in agreement with the expected flow behaviour. Indeed, assume that the hydraulic conductivity decreases over the area $\Omega$. Then head losses increase, and the hydraulic head increases upstream of the perturbed area and decreases downstream. The contour lines of $\eta_{K}$ are defined by a constant $r_{1} / r_{2}$ ratio. The sensitivity contour lines are thus circles, the centre of which lies on the $y$-axis and is converging at the source 1 or at the source 2 for disminishing radius (see Figure 4a). Note that the sensitivity propagates mainly in the flow direction. The sensitivity propagates upstream and downstream with the same intensity, consequently the sensitivity pattern of the hydraulic head is symmetric with respect to the $x$-axis.

The sensitivities $\nu_{K}$ and $\varpi_{K}$ of the longitudinal and transverse velocities can be derived using the results of Section 2.2

$$
\begin{aligned}
\nu_{K} & =\frac{2 L^{2}}{3 \pi} \frac{\partial H}{\partial y} \frac{x^{2}+(2 L / 3)^{2}-y^{2}}{r_{1}^{2} r_{2}^{2}} \\
\varpi_{K} & =-\frac{4 L^{2}}{3 \pi} \frac{\partial H}{\partial y} \frac{x y}{r_{1}^{2} r_{2}^{2}}
\end{aligned}
$$

Unlike the sensitivity of the hydraulic head, the sensitivities of the transverse and longitudinal velocities propagate in the transverse direction. Figures $4 \mathrm{~b}$ and $4 \mathrm{c}$ show typical contour lines for $\nu_{K}$ and $\varpi_{K}$ respectively. The contour lines of the sensitivity of the longitudinal flow velocity have the shape of a cross, directed along the flow. The sensitivity of the transverse component of the flow velocity is also cross-shaped, but its branches are diagonal to the flow. This can be interpreted physically as follows. Assume that the hydraulic conductivity of the area $\Omega$ is lower than that of the remainder of the model. Then 


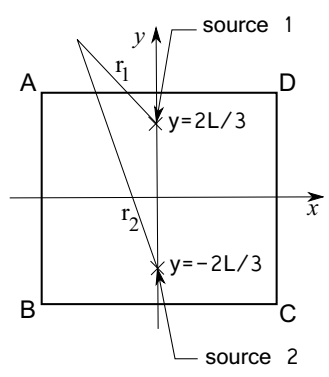

Figure 2: Equivalent source configuration for $\varepsilon_{K}$ in the confined case.

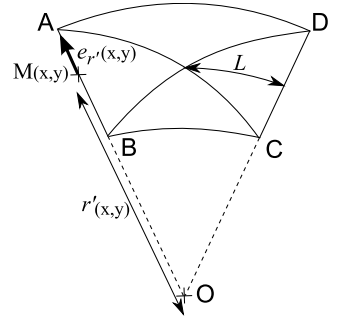

Figure 3: Definition sketch for for $\varepsilon$, non parallel flow case (plan view). The point $O$ is the convergence point of the flow path lines, the point $\mathrm{M}$ has coordinate $(\mathrm{x}, \mathrm{y})$ in the cartesian frame, $r^{\prime}$ is the distance from $\mathrm{M}$ to $\mathrm{O}$ and $e_{r}$ is the radial unit vector at $M$.

the flow path lines tend to pass round the low-conductivity area, leading to diverging flow pattern upstream from the perturbed area, and converging flow pattern downstream. A small, positive $x$-velocity appears to the upstream left and downstream right from the perturbed area, while a small, negative $x$-velocity appears to the upstream right and downstream left (Figure 4c). Besides, the flow is slowed down upstream and downstream from the low-conductivity area, while it is speeded up in the transverse direction to the flow (Figure 4b).
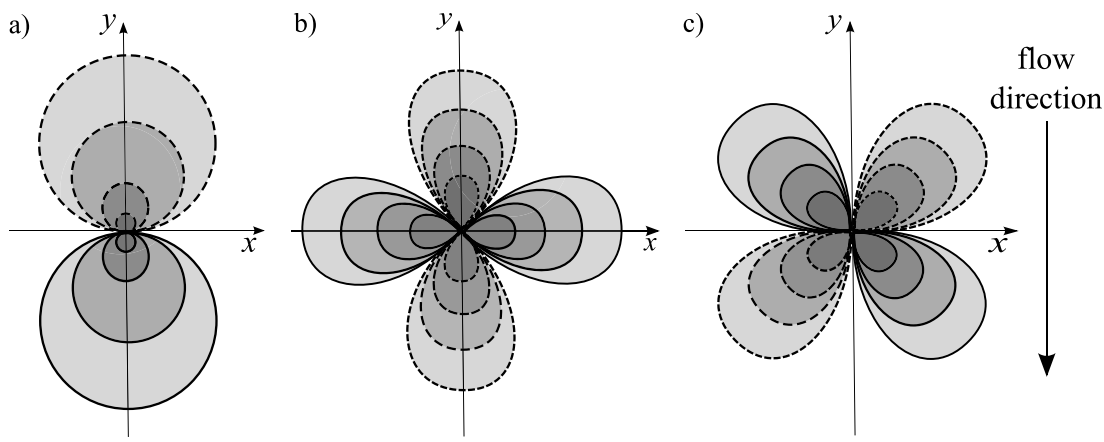

Figure 4: Parallel, steady-state confined flow : case of a perturbation in the hydraulic conductivity. Typical far field behaviour for the contour lines of : a) the hydraulic head sensitivity, b) the longitudinal velocity sensitivity, c) the transverse velocity sensitivity. Darker zones indicate higher absolute value. Dashed and solid lines indicate negative and positive values respectively.

\subsection{Influence of a perturbation in the aquifer thickness for parallel flow}

For confined aquifers, a modification of the aquifer thickness amounts to a modification of the aquifer bedrock elevation. The sensitivity source term generated by a perturbation in the aquifer thickness is equal to that generated by a perturbation in the hydraulic conductivity, multiplied by a factor $K / e$. The sensitivity field generated by a perturbation 
in the aquifer thickness can thus be derived by analogy with the results of Section 3.1

$$
\begin{aligned}
\eta_{e} & =\frac{L}{2 \pi e} \frac{\partial H}{\partial y} \ln \left(\frac{r_{1}}{r_{2}}\right) \\
\nu_{e} & =\frac{\partial H}{\partial y} \frac{2 L^{2} K}{3 \pi e} \frac{x^{2}+(2 L / 3)^{2}-y^{2}}{r_{1}^{2} r_{2}^{2}} \\
\varpi_{e} & =-\frac{4 L^{2} K}{3 \pi e} \frac{\partial H}{\partial y} \frac{x y}{r_{1}^{2} r_{2}^{2}}
\end{aligned}
$$

The sensitivity patterns induced by a perturbation in the aquifer thickness are the same as previously seen for a perturbation of the hydraulic conductivity. Note that

$$
K \eta_{K}=e \eta_{e}
$$

Equation (22) means that a given relative variation in $K$ has the same effect on the hydraulic head and the flow velocities as the same relative variation in $e$.

\subsection{Influence of a perturbation in the recharge for parallel flow}

It stems from equation (5c) that the source term generated by a perturbation in the recharge reduces to $\varepsilon(x, y)$. Its integral over the perturbed area is $4 L^{2} / 3$. For far-field behaviour, this source configuration is equivalent to a point source of value $4 L^{2} / 3$ located at $(0,0)$. The field generated by a point source is known from the theory of potential flow [32] to be

$$
\eta_{R}=-\frac{2 L^{2}}{3 \pi K e} \ln \left(\frac{r}{d}\right)
$$

where $d$ is the distance for which the sensitivity is zero. For an infinite domain, $d$ must be fixed arbitrarily. In real-world applications, $d$ depends on the boundary conditions. The contour lines of the sensitivity are circles centred on the perturbation. Figure 5 shows typical sensitivity patterns for $\eta_{R}$. Note that the value of the sensitivity is proportional to the inverse of the aquifer thickness and hydraulic conductivity, which means that the estimate of the aquifer recharge is all the more important as the aquifer is thin or little conductive. Denoting by $\delta$ the value below which $\eta$ is considered negligible, equation (23) leads to

$$
r_{(\eta=\delta)}=d \exp \left(-\frac{3 \pi \delta}{2 L^{2}} K e\right)
$$

Equation (24) shows that for a given $d$ and $L$, the distance beyond which the sensitivity can be considered negligible decreases exponentially with the aquifer transmissivity $K e$.

The sensitivities $\rho_{R}$ and $\theta_{R}$ of the radial and tangential flow velocities are

$$
\begin{aligned}
\rho_{R} & =\frac{2 L^{2}}{3 \pi e} \frac{1}{r} \\
\theta_{R} & =0
\end{aligned}
$$

The sensitivity propagates radially.

\subsection{Influence of a perturbation in the flow parameters for non-parallel flow}

The sensitivity to the recharge is independent from the hydraulic head field, therefore non-parallel flow yields no change in the sensitivity patterns obtained in Section 3.3. The case of a perturbation in the hydraulic conductivity or in the aquifer thickness is different. Since the gradient of the hydraulic head gradient cannot be considered constant over the perturbed area, the source term changes. 


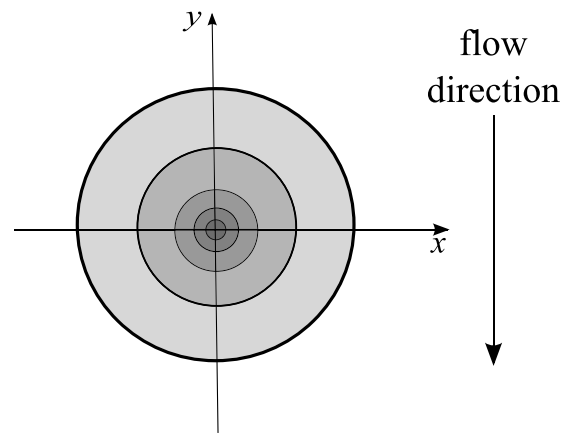

Figure 5: Parallel, steady-state confined flow : case of a perturbation in the recharge rate. Typical far field behaviour of the hydraulic head sensitivity contour lines. Darker zones indicate higher absolute value. Solid lines indicate positive values.

Consider the case of a perturbation in the hydraulic conductivity. Assume that the flow can be approximated as radial over the perturbed area. The flow velocity may thus be expressed as $v=-Q /\left(2 \pi e r^{\prime}\right) e_{r^{\prime}}$, where $r^{\prime}$ is the distance to the convergence point of the flow path lines,$e_{r^{\prime}}$ is the radial unit vector and $Q$ is positive for converging flow and negative for diverging flow (see definition sketch in Figure 3). Assuming that $K$ is a constant over $\Omega$, the source term may be expressed as

$$
\rho_{K}=\frac{Q}{2 \pi K}\left[\rho_{1}+\rho_{2}\right]
$$

where

$$
\begin{aligned}
& \rho_{1}=-\frac{\varepsilon}{{r^{\prime}}^{2}} \\
& \rho_{2}=\frac{1}{r^{\prime}} \frac{\partial \varepsilon}{\partial r^{\prime}}
\end{aligned}
$$

When $L \ll r^{\prime}, \rho_{1}$ is negligible compared to $\rho_{2}$ and the variations of $r^{\prime}$ over $\Omega$ can be neglected. The source term then reduces to $\rho_{K}=\left[Q /\left(2 \pi K r_{o}^{\prime}\right)\right] \partial \varepsilon / \partial r^{\prime}$, where $r_{o}^{\prime}$ is the distance between the perturbation and the convergence point of the flow path lines. This source configuration is equivalent for far-field behaviour to a doublet of sources, as in the parallel flow case. It yields a symmetric hydraulic head sensitivity pattern with respect to the $x$-axis, with negative sign upstream of the perturbed area, and positive sign downstream. The second-order terms yields an increase of the absolute sensitivity values in the converging flow path lines direction (see demonstration in Appendix A.2). Indeed, the perturbation in the hydraulic heads is expected to be more important as the flow section decreases.

The case of a perturbation in the aquifer thickness is similar to that of a perturbation in the hydraulic conductivity.

\section{Sensitivity properties for unconfined aquifers}

The theoretical developments are carried out using the same assumptions as in Section 3 and assuming that the aquifer bedrock elevation is constant. The values of the hydraulic head and bedrock elevation at the centre of the perturbed area are denoted by $H_{o}$ and $z_{o}$. 


\subsection{Influence of a perturbation in the hydraulic conductivity for parallel flow}

The detailed derivation of the source term is provided in Appendix A.3. The far field sensitivity source configuration is equivalent to the superposition of a doublet of sources of intensity $-L(H-z)_{o}(\partial H / \partial y)_{o}$ (point source 1) and $+L(H-z)_{o}(\partial H / \partial y)_{o}$ (point source 2 ) located at respectively $(0,2 L / 3)$ and $(0,-2 L / 3)$ (see Figure 2$)$. The field generated by this equivalent source configuration is known from the theory of potential flow [32] to be

$$
\eta_{K}=\frac{L}{2 \pi K}\left(\frac{\partial H}{\partial y}\right)_{o} \ln \left(\frac{r_{1}}{r_{2}}\right) \frac{H_{o}-z_{o}}{H-z}
$$

where $r_{1}$ and $r_{2}$ are the distances to the equivalent sources 1 and 2 respectively. The term $\left(H_{o}-z_{o}\right) /(H-z)$ is an advection term. As its absolute value is larger dowstream than upstream for a given distance to the perturbation, the contour lines are shifted downstream. The resulting sensitivity pattern is shown in Figure 6a.

The sensitivities $\nu_{K}$ and $\varpi_{K}$ of the longitudinal and transverse flow velocities are

$$
\begin{aligned}
\nu_{K} & =-\frac{L}{\pi}\left(\frac{\partial H}{\partial y}\right)_{o}\left[\frac{4 L}{3} \frac{x^{2}+4 L^{2} / 9-y^{2}}{r_{1}^{2} r_{2}^{2}}-\frac{1}{2(H-z)} \frac{\partial H}{\partial y} \ln \left(\frac{r_{1}}{r_{2}}\right)\right] \frac{H_{o}-z_{o}}{H-z} \\
\varpi_{K} & =-\frac{2 L^{2}}{3 \pi}\left(\frac{\partial H}{\partial y}\right)_{o} \frac{x y}{r_{1}^{2} r_{2}^{2}} \frac{H_{o}-z_{o}}{H-z}
\end{aligned}
$$

As an effect of advection, the contour lines of the sensitivity of the flow transverse velocity are shifted downstream. The sensitivity of the longitudinal flow velocity is made of two terms with opposite effects. The first term on the right-hand side of equation (29a) yields sensitivity patterns similar to those obtained in the confined case, but shifted downstream. The second term on the right-hand side of equation (29a) has negative sign upstream from the perturbation, and positive sign downstream. Its effect is a decrease of the algebraic sensitivity value upstream from the perturbed area, and an increase downstream. The resulting sensitivity patterns are shown in Figures $6 \mathrm{~b}$ and $6 \mathrm{c}$.
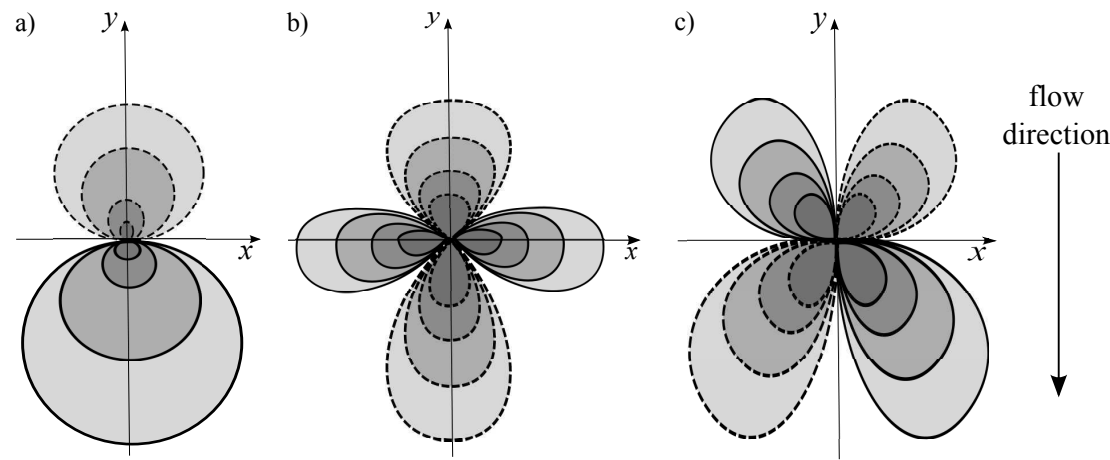

Figure 6: Parallel, steady-state unconfined flow : case of a perturbation in the hydraulic conductivity. Typical far field behaviour for the contour lines of : a) the hydraulic head sensitivity, b) the longitudinal velocity sensitivity, c) the transverse velocity sensitivity. Darker zones indicate higher absolute value. Dashed and solid lines indicate negative and positive values respectively. The contour lines deformation has been exaggerated for a better understanding.

\subsection{Influence of a perturbation in the bedrock elevation for parallel flow}

Assume that the flow is parallel, directed along the $y$-axis over the perturbed area. Also assume that the hydraulic head gradient is constant over the perturbed area. Then the calculation of the source term generated by a perturbation in the bedrock elevation is 
similar to that of the source term generated by a perturbation in the hydraulic conductivity. The sensitivity field generated by a perturbation in the bedrock elevation can thus be derived by analogy with the results of Section 4.1

$$
\eta_{z}=-\frac{L}{2 \pi}\left(\frac{\partial H}{\partial y}\right)_{o} \ln \left(\frac{r_{1}}{r_{2}}\right) \frac{1}{H-z}
$$

The sensitivities $\nu_{z}$ and $\varpi_{z}$ of the longitudinal and transverse flow velocities are

$$
\begin{aligned}
\nu_{z} & =-\frac{L K}{\pi}\left(\frac{\partial H}{\partial y}\right)_{o}\left[\frac{4 L}{3} \frac{x^{2}+4 L^{2} / 9-y^{2}}{r_{1}^{2} r_{2}^{2}}-\frac{1}{2(H-z)} \frac{\partial H}{\partial y} \ln \left(\frac{r_{1}}{r_{2}}\right)\right] \frac{1}{H-z} \\
\varpi_{z} & =-\frac{2 L^{2} K}{3 \pi}\left(\frac{\partial H}{\partial y}\right)_{o} \frac{x y}{r_{1}^{2} r_{2}^{2}} \frac{1}{H-z}
\end{aligned}
$$

\subsection{Influence of a perturbation in the recharge for parallel flow}

The sensitivity of the hydraulic head to the recharge can be obtained directly by analogy with the confined case

$$
\eta_{R}=-\frac{2 L^{2}}{3 \pi K} \ln \left(\frac{r}{d}\right) \frac{1}{H-z}
$$

Compared to the confined case, the sensitivity contour lines are advected downstream (see Figure $7 b)$.

The sensitivities $\rho_{R}$ and $\theta_{R}$ of the radial and tangent flow velocities are

$$
\begin{aligned}
& \rho_{R}=\frac{2 L^{2}}{3 \pi} \frac{1}{H-z}\left[\frac{1}{r}+\ln \left(\frac{r}{d}\right) \frac{\partial(H-z)}{\partial y} \frac{\cos \theta}{H-z}\right] \\
& \theta_{R}=-\frac{2 L^{2}}{3 \pi} \ln \left(\frac{r}{d}\right) \frac{\partial(H-z)}{\partial y} \frac{\sin \theta}{(H-z)^{2}}
\end{aligned}
$$

where $\theta$ is the angle coordinate with respect to the $y$-axis. The sensitivity of the radial flow velocity is made of two terms. The first term $2 L^{2} /[3 \pi r(H-z)]$ is independent from the angular coordinate $\theta$. It is equal to the sensitivity of the radial flow velocity derived in the confined case, multiplied by a factor $1 /(H-z)$. The effect of the $1 /(H-z)$ factor is to advect the sensitivity of the radial flow velocity downstream. The second term of the sensitivity of the radial flow velocity and the sensitivity of the tangential flow velocity $\theta_{R}$ are better understood using Cartesian coordinates. Using Cartesian coordinates, the sensitivities $\nu_{R}$ and $\varpi_{R}$ of the longitudinal and transverse flow velocities are (see Appendix A.4 for details)

$$
\begin{aligned}
\nu_{R} & =\frac{2 L^{2}}{3 \pi} \frac{y}{(H-z) r^{2}}+\frac{2 L^{2}}{3 \pi(H-z)^{2}} \frac{\partial(H-z)}{\partial y} \ln \left(\frac{r}{d}\right) \\
\varpi_{R} & =\frac{2 L^{2}}{3 \pi} \frac{x}{(H-z) r^{2}}
\end{aligned}
$$

The first term on the right-hand side of each equation corresponds to $2 L^{2} /[3 \pi r(H-z)]$ in radial coordinates. The effect of the term $\left[\left(2 L^{2}\right) /\left(3 \pi(H-z)^{2}\right)\right] \ln (r / d) \partial(H-z) / \partial y$ is to decrease the longitudinal velocity sensitivity upstream of the perturbation, and to increase it downstream (see details in Appendix A.4). This can be explained physically by the fact that an increase in the recharge causes the water level to increase upstream of the perturbed area (and to decrease downstream). This leads to an increase of the algebraic value of the hydraulic head gradient upstream of the perturbed area (and to a decrease downstream), which in turns causes the flow velocity to decrease upstream of the perturbed area (and to increase downstream) (see Figure 7a). 
a)

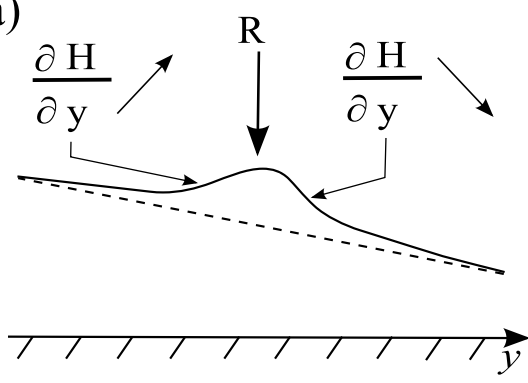

b)

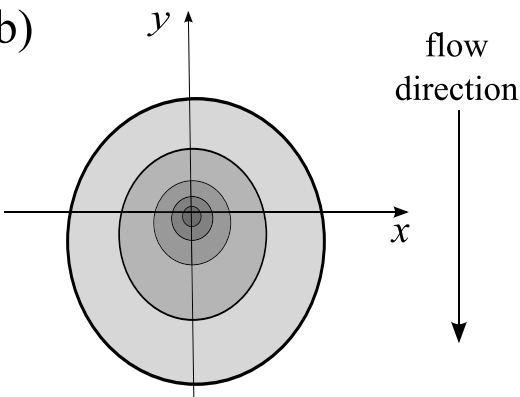

Figure 7: Parallel, steady-state unconfined flow : case of a perturbation in the recharge rate. a) Longitudinal head profile crossing the perturbed area. Dashed line : unperturbed head profile, solid line : perturbed head profile. An increase in the recharge causes the algebraic value of the hydraulic head gradient to increase upstream of the perturbed area, and to decrease downstream, b) Typical far field behaviour of the hydraulic head sensitivity contour lines. Solid lines indicate positive values. The contour lines deformation has been exaggerated in order to ease the understanding.

\subsection{Influence of a perturbation in the flow parameters for non-parallel flow}

Non-parallel flow modifies the sensitivities established in the previous sections in two different ways. The sensitivity to all parameters is proportional to the inverse of the hydraulic head, which accounts for the advection of the perturbation in the flow direction. The variation in the hydraulic head gradient over the perturbed area also influences the source term of the sensitivities to the hydraulic conductivity and to the aquifer bottom level elevation, as in the confined case.

Consider the case of a perturbation in the hydraulic conductivity, and assume that the flow can be approximated locally with a radial flow field. The flow velocity may thus be expressed as $v=-Q /\left(2 \pi r^{\prime}(H-z)\right) e_{r^{\prime}}$. The source term of the sensitivity to the hydraulic conductivity becomes $\rho_{K}=\nabla\left[Q \varepsilon /\left(2 \pi K r^{\prime}\right) e_{r^{\prime}}\right]$. This expression is very close to that of the confined case, so that the results of Section 3.4 can be adapted directly. The sensitivity patterns are the same as in the parallel flow case, but multiplied by a factor $1 /(H-z)$. The effects of the distortion by the hydraulic head field and of the variation of the intensity of the source term over the perturbed area may be opposed.

Consider now the case of a perturbation in the aquifer bedrock elevation. Under a radial flow approximation, the source term of the sensitivity can be written as

$$
\rho_{z}=-\nabla\left[\frac{Q \varepsilon}{2 \pi(H-z) r^{\prime}} e_{r^{\prime}}\right]
$$

The difference with the previous case lies in the coefficient $1 /(H-z)$, that contributes to increase the sensitivity downstream of the perturbation.

\section{Boundary conditions}

\subsection{Sensitivity to boundary conditions}

Boundary conditions along a boundary $b$ may be written in general form as

$$
f\left(x_{b}, y_{b}, \phi_{b}, H_{b}\right)=0
$$

where the function $f$ is known and the subscript $b$ refers either to the value of the parameter or to the variable along the boundary. Differentiating equation (36) with respect to the parameter $\phi$ leads to the following equation

$$
\frac{\partial f}{\partial H} \frac{d H}{d \phi_{b}}+\frac{\partial f}{\partial \phi_{b}}=0
$$


which in turn leads to the sensitivity boundary condition

$$
\frac{\partial f}{\partial H} \eta_{\phi_{b}}+\frac{\partial f}{\partial \phi_{b}}=0
$$

where the term $\partial f / \partial \phi_{b}$ is known from the expression of the boundary conditions.

The case of the most commonly employed boundary conditions is detailed hereafter. Prescribed head conditions may be written as

$$
H-H_{b}=0
$$

where $H_{b}$ is the value of the hydraulic head along the boundary. Note that equation (39) is only valid along the boundary. In this writing, $f$ is defined as

$$
f\left(H, H_{b}\right)=H-H_{b}
$$

and the perturbed parameter is $H_{b}$

$$
\begin{aligned}
\phi_{b} & =H_{b} \\
\partial f / \partial H_{b} & =-\varepsilon \\
\partial f / \partial H & =1
\end{aligned}
$$

Equation (38) thus becomes

$$
\eta_{H_{b}}=\varepsilon
$$

Similarly, flux conditions and head-flux relationships may be written as

$$
\begin{aligned}
-K e \nabla H & =F_{b} \\
-K H \nabla H & =F_{b} \\
-K e \nabla H & =\lambda H \\
-K H \nabla H & =\lambda H
\end{aligned}
$$

$$
\text { (prescribed flux, confined flow) }
$$

(head-flux relationship, confined flow)

where $F_{b}$ is the flux prescribed along the boundary and $\lambda$ is the fluid transfer coefficient (leakage parameter). Differentiating equations (43a) to (43d) with respect to $F_{b}$ and $\lambda$ respectively leads to

$$
\begin{array}{rlr}
-K e \nabla \eta_{F_{b}} & =\varepsilon & \text { (prescribed flux, confined flow) } \\
-K \eta_{F_{b}} \nabla H-K H \nabla \eta_{F_{b}} & =\varepsilon & \text { (prescribed flux, unconfined flow) } \\
-K e \nabla \eta_{\lambda} & =\varepsilon H+\lambda \eta_{\lambda} & \text { (head-flux relationship, confined flow) } \\
-K \eta_{\lambda} \nabla H-K H \nabla \eta_{\lambda} & =\varepsilon H+\lambda \eta_{\lambda} & \text { (head-flux relationship, unconfined flow) }
\end{array}
$$

Prescribing boundary conditions thus amounts to prescribing the boundary value of the sensitivity or sensitivity gradient, or a relationship between the sensitivity and the sensitivity gradient, depending on the nature of the boundary condition.

Inside the model, the sensitivity obeys equation (7) (confined aquifer) or (16) (unconfined aquifer)

$$
\begin{aligned}
\nabla\left(K e \nabla \eta_{\phi}\right) & =0 & & \text { (confined flow) } \\
\nabla\left(K(H-z) \nabla \eta_{\phi}\right) & =0 & & \text { (unconfined flow })
\end{aligned}
$$

The solution of this equation depends on the boundary conditions fixed by equation (38). There is no analytical solution in the two-dimensional general case. 


\subsection{One-dimensional case}

Assume that the problem is one-dimensional. Denote by $T$ the transmissivity $K e$ (confined case) or $K(H-z)$ (unconfined case). Then equations (45a) and (45b) imply that

$$
T \frac{\partial \eta_{\phi}}{\partial n}=A
$$

where $A$ is a constant that depends on the boundary conditions. The hydraulic head sensitivity gradient $\partial \eta_{\phi} / \partial n$ is proportional to the inverse of the transmissivity.

If the transmissivity is uniform, then the hydraulic head sensitivity decreases linearly inside the model. Assume that the aquifer transmissivity is not homogeneous. Equation (46) implies that the hydraulic head sensitivity gradient in a given region is proportional to the inverse of the transmissivity.

Consider the case of a fixed head boundary condition. The value of the hydraulic head sensitivity at the perturbed boundary is equal to 1 , regardless of the transmissivity value (see Section 5.1). The normal gradient of the hydraulic head sensitivity across the model depends on the transmissivity value based on equation (46). The value of the hydraulic head sensitivity at a given distance from the perturbed boundary thus depends on the average transmissivity value between the perturbed boundary and the location at which the sensitivity is investigated. The error stemming from wrongly specified head boundary condition will thus be minimized if the boundary lies in a low transmissivity area.

The case of flux boundary conditions and head-flux relationships is different. As for hydraulic head boundary conditions, a change in the transmissivity leads to a change in the hydraulic head sensitivity gradient. Yet the change in the hydraulic head sensitivity gradient is counterbalanced by a change in the hydraulic head sensitivity value at the perturbed boundary (see Appendix B). As a consequence, the error or the uncertainty stemming from wrongly specified or uncertain boundary condition is not minimized if the boundary lies in a region with low transmissivity. In contrast, the uncertainty in the simulated hydraulic head is minimized for low average transmissivity between the area over which the hydraulic head is investigated and a well-known prescribed head condition.

The sensitivity of the flow velocity is related to the hydraulic head sensitivity based on equation (14). Equation (46) can thus be recast as

$$
\begin{array}{lr}
\omega_{\phi}=-A / e & \text { (confined flow) } \\
\omega_{\phi}=-A /(H-z) & \text { (unconfined flow) }
\end{array}
$$

which means that the sensitivity of the flow velocity is proportional to the inverse of the aquifer thickness.

\section{Computational examples}

\subsection{Parallel flow in a confined aquifer: sensitivity to $K$ and $R$}

The present test case aims at checking that the theoretical results of Section 3 match the experimental sensitivities computed under confined parallel flow conditions. Note that as the analytical expressions established in Section 3 and 4 are approximate solutions of the sensitivity equation, the difference between the numerical results and the approximate farfield sensitivity is not expected to necessarily converge to zero. Two simulations are carried out. In the first simulation, the investigated parameter $\phi$ is uniform while in the second simulation, it is perturbed by a small amount $\phi_{o}$ over a square region of size $L$. The empirical sensitivity of the flow variable is computed as the ratio of the difference between the two simulation results to the perturbation $\phi_{o}$. The two-dimensional groundwater flow equations are solved over a square domain of size $D$ using the finite element FEFLOW numerical code 
$[33,34]$. The lateral boundaries $(x= \pm D / 2)$ are no flow boundaries. A constant hydraulic head is prescribed at the upstream $(y=D / 2)$ and downstream $(y=-D / 2)$ boundaries of the domain, so as to allow steady state parallel flow. Note that in the following simulations, the flow is directed in the negative $y$ direction. The resulting sensitivity patterns are drawn over a square domain of size $d \ll D$ in order to eliminate artefacts due to the boundaries (see definition sketch in Figure 8). The model characteristics are summarized in Table 1.

Figures 9a to $9 \mathrm{c}$ show typical sensitivity patterns generated by a perturbation in the hydraulic conductivity (see test case parameters in Table 2). The resulting sensitivity patterns match the results of Section 3.1 (Figure 4).

Figure 10 shows the hydraulic head sensitivity pattern generated by a perturbation in the recharge (see test case parameters in Table 2). The experimental sensitivity pattern is in agreement with the results of Section 3.3 (Figure 5).

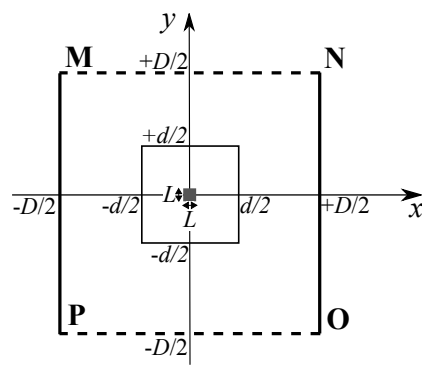

Figure 8: Definition sketch for the model used in application examples 6.1 (parallel flow in a confined aquifer) and 6.2 (parallel flow in an unconfined aquifer). The simulations are run in a square domain of size $D$. The hydraulic head is fixed along the borders (MN) and (OP). No-flux boundary conditions are prescribed along the borders (NO) and (PM). The parameters are perturbed over a square area of length $L$ at the centre of the domain (grayed area). The resulting sensitivity patterns are drawn over a square domain of size $d$ centred on the perturbed area.
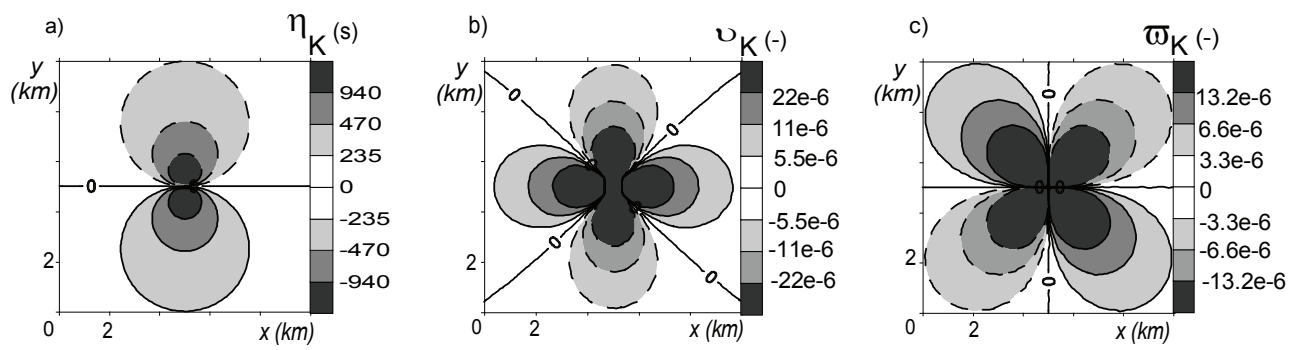

Figure 9: Parallel, steady-state confined flow. Case of a perturbation in the hydraulic conductivity : a) sensitivity of the hydraulic head, b) sensitivity of the longitudinal flow velocity, c) sensitivity of the transverse flow velocity. Dashed and solid lines indicate negative and positive values respectively. The simulation parameters are given in Table 1 and Table 2.

\subsection{Parallel flow in an unconfined aquifer: sensitivity to $K$ and $R$}

The present test case aims at checking that the theoretical results of Section 4 match the experimental sensitivities computed under unconfined parallel flow conditions. The methodology is the same as in Section 6.1. The test case parameters are given in Table 3 and 4.

Figures 12a to 12c show typical sensitivity patterns generated by a perturbation of the hydraulic conductivity. Compared to the confined case, the sensitivity patterns are advected 


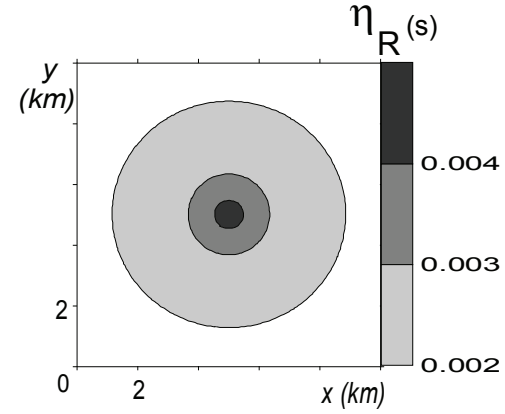

Figure 10: Parallel, steady-state confined flow. Case of a perturbation in the recharge rate : hydraulic head sensitivity. The simulation parameters are given in Table 1 and Table 2.

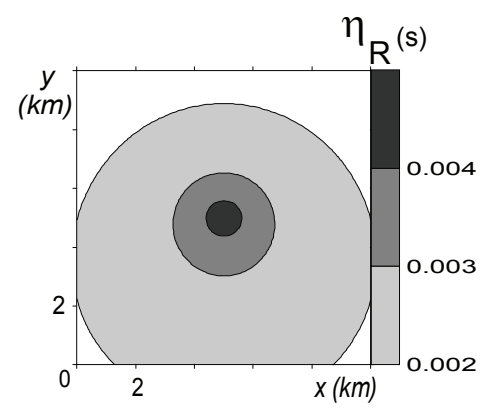

Figure 11: Parallel, steady-state unconfined flow. Case of a perturbation in the recharge rate : hydraulic head sensitivity. The simulation parameters are given in Table 3 and Table 4 .

\begin{tabular}{lll}
\hline Symbol & Meaning & Value \\
\hline$D$ & $\begin{array}{l}\text { Length of the } \\
\text { simulation domain } \\
\text { Length of the results } \\
\text { visualization area }\end{array}$ & $10 \mathrm{~km}$ \\
$L$ & $\begin{array}{l}\text { Length of the perturbed area } \\
H_{M N}\end{array}$ & $\begin{array}{l}\text { Hydraulic head prescribed } \\
\text { along the (MN) border }\end{array}$ \\
$H_{O P}$ & $\begin{array}{l}\text { Hydraulic head prescribed } \\
\text { along the (OP) border }\end{array}$ & $10 \mathrm{~m}$ \\
\hline
\end{tabular}

Table 1: Model characteristics for the application examples described in Section 6.1 (steady-state confined parallel flow). (MP) and (NO) are no-flow boundaries.

\begin{tabular}{lll}
\hline Symbol & Meaning & Value \\
\hline$K$ & Hydraulic conductivity & $10^{-4} \mathrm{~m} / \mathrm{s}$ \\
$R$ & Recharge rate & $0 \mathrm{~m} / \mathrm{s}$ \\
$e$ & Aquifer thickness & $78.2 \mathrm{~m}$ \\
$k_{o}$ & $\begin{array}{l}\text { Perturbation in the } \\
\text { hydraulic conductivity }\end{array}$ & $-2.510^{-5} \mathrm{~m} / \mathrm{s}$ \\
$r_{o}$ & $\begin{array}{l}\text { Perturbation in the } \\
\text { recharge rate }\end{array}$ & $5.810^{-9} \mathrm{~m} / \mathrm{s}$ \\
\hline
\end{tabular}

Table 2: Parallel, steady-state confined flow. Parameters for the application example described in Section 6.1 : case of a perturbation in the hydraulic conductivity or in the recharge rate. 
in the direction of the flow, as predicted in Section 4.1 (Figure 6). Remember that contour lines deformation on Figure 6 has been exaggerated for comprehension purpose. Figure 11 shows the hydraulic head sensitivity pattern generated by a perturbation in the recharge. The deformation due to the advection is clearly visible. The experimental sensitivity pattern matches the results of Section 4.3 (Figure 7).
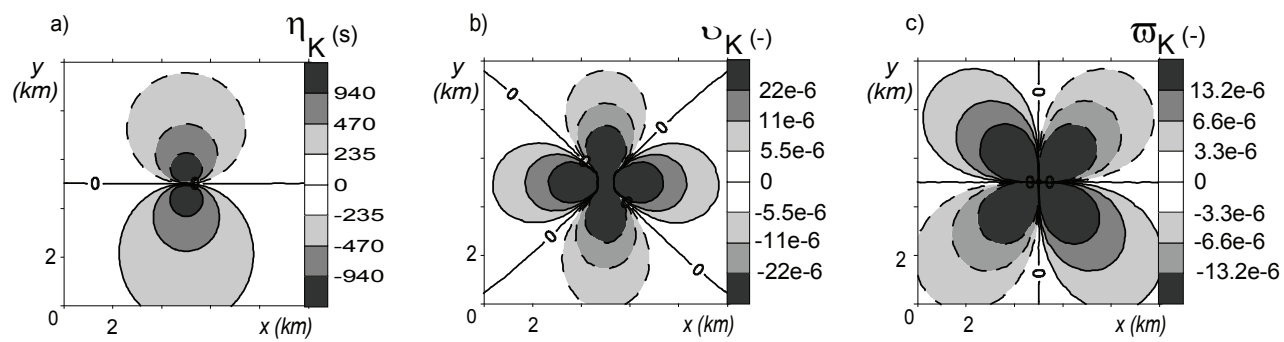

Figure 12: Parallel, steady-state unconfined flow. Case of a perturbation in the hydraulic conductivity : a) sensitivity of the hydraulic head, b) sensitivity of the longitudinal flow velocity, c) sensitivity of the transverse flow velocity. Dashed and solid lines indicate negative and positive values respectively. The simulation parameters are given in Table 3 and Table 4 .

\subsection{Non-parallel flow in a mixed confined-unconfined aquifer : sensitivity to $K$ and $R$}

The present test case aims at checking the validity of the theoretical sensitivity patterns derived in Section 4 in a real-site geometry. With this purpose, the sensitivity patterns generated by a perturbation in the hydraulic conductivity or in the recharge are investigated under non parallel flow conditions over a schematic representation of the Lez karst aquifer system (Hérault, France).

The Lez aquifer system is developed mainly in karstified Jurassic to late Cretaceous limestones, with a thickness ranging from 650 to $1100 \mathrm{~m}$ [35]. Its main outlet is the Lez spring, that supplies the city of Montpellier with water. The aquifer system is bounded by the Hérault and Vidourle rivers at its western and eastern sides, and by impervious structural boundaries at its northern and southern sides [36] (see Figure 13). The aquifer limestones outcrops over half of the aquifer surface. In the following, no account is taken of the karst conduit network. A flux boundary condition is set at the location of the pumping station (Lez spring). The pumping rate $Q$ is taken equal to the mean of the annual discharge under natural regime. No-flow boundaries are used to represent the northern and southern borders. At the eastern and western borders, the hydraulic head is prescribed and taken equal to the average annual stream stage. The recharge $R$ is assumed uniform. A 3-D geological model of the aquifer has been constructed based on

\begin{tabular}{lll}
\hline Symbol & Meaning & Value \\
\hline$D$ & $\begin{array}{l}\text { Length of the simulation } \\
\text { domain }\end{array}$ & $40 \mathrm{~km}$ \\
$d$ & $\begin{array}{l}\text { Length of the results } \\
\text { visualization area }\end{array}$ & $10 \mathrm{~km}$ \\
$L$ & $\begin{array}{l}\text { Length of the perturbed area } \\
H_{M N}\end{array}$ & $\begin{array}{l}\text { Hydraulic head prescribed } \\
\text { along the (MN) border }\end{array}$ \\
$H_{O P}$ & $\begin{array}{l}\text { Hydraulic head prescribed } \\
\text { valong the (OP) border }\end{array}$ & $110 \mathrm{~m}$ \\
\hline
\end{tabular}

Table 3: Model characteristics for the application examples described in Section 6.2 (steady-state unconfined parallel flow). (MP) and (NO) are no-flow boundaries. 


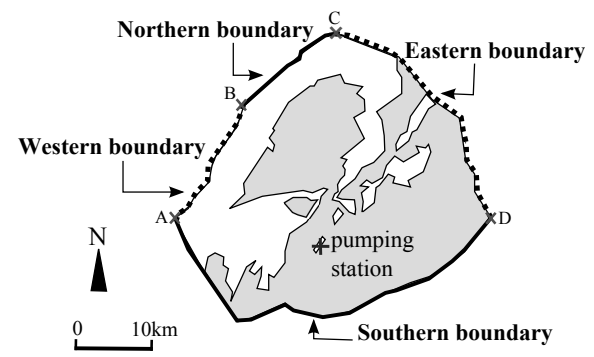

Figure 13: Definition sketch for the Lez model. The confined portion of the aquifer is grayed out.

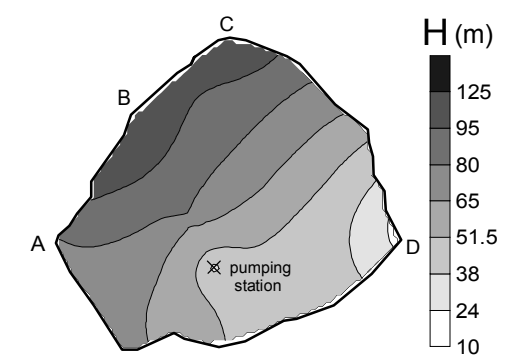

Figure 14: Steady state piezometric map of the Lez model.

lithological descriptions [36], regional geological maps [37], available cross-sections [38, 39] and available stratigraphic logs, nine of which intersect the whole stratigraphic serie. The aquifer hydraulic conductivity $K$ is assumed uniform, yet the perturbed aquifer bedrock geometry yields non-uniform aquifer transmissivity.

Experimental sensitivities are computed using the same methodology as in Section 6.1. The parameters are given in Table 5. The steady-state piezometry and transmissivity maps are presented in Figures 14 and 15.

Figures $17 \mathrm{a}$ to $17 \mathrm{c}$ show the sensitivity patterns generated by a perturbation in the hydraulic conductivity. Compared to the parallel flow case, the absolute value of the sensitivity is greater in the converging flow path direction. Figure 16 shows the hydraulic head sensitivity pattern generated by a perturbation in the recharge. The sensitivity contour lines are not fully circular due to the influence of the boundaries.

\subsection{Non-parallel flow in a mixed confined-unconfined aquifer : sensitivity to $K$ under transient conditions}

The present test case aims at checking the validity under transient flow conditions of the theoretical sensitivity patterns derived in Section 4. With this purpose, the sensitivity pattern generated by a perturbation in the hydraulic conductivity is investigated under transient conditions over a schematic representation of the Lez karst aquifer system. Experimental sensitivities are computed using the same methodology as in Section 6.1. Test case parameters are given in Table 6. The initial hydraulic heads are obtained from the steady-state simulation in Section 6.3. The recharge rate is interpolated from daily rainfall data recorded at 13 raingauge stations distributed over the Lez basin from the 26th of november 2002 (day 1) to the 18th of february 2003 (day 85) (see Figure 18). The rainfall event from day 15 to day 17 is centred over the western part of the Lez basin (see cumulated

\begin{tabular}{lll}
\hline Symbol & Meaning & Value \\
\hline$K$ & Hydraulic conductivity & $10^{-4} \mathrm{~m} / \mathrm{s}$ \\
$R$ & Recharge rate & $0 \mathrm{~m} / \mathrm{s}$ \\
$z$ & $\begin{array}{l}\text { Aquifer bedrock elevation } \\
k_{o}\end{array}$ & $\begin{array}{l}\text { Perturbation in the } \\
\text { hydraulic conductivity }\end{array}$ \\
$r_{o}$ & $\begin{array}{l}\text { Perturbation in the } \\
\text { recharge rate }\end{array}$ & $5.810^{-5} \mathrm{~m} / \mathrm{s}$ \\
$H(0,0)$ & $\begin{array}{l}\text { Hydraulic head at the centre } \\
\text { of the perturbed area }\end{array}$ & $78.2 \mathrm{~m}$ \\
$\nabla H(0,0)$ & $\begin{array}{l}\text { Hydraulic head gradient at the } \\
\text { centre of the perturbed area }\end{array}$ & $1.9 \mathrm{~mm} / \mathrm{m}$ \\
& &
\end{tabular}

Table 4: Parallel, steady-state unconfined flow. Parameters for the application example described in Section 6.2 : case of a perturbation in the hydraulic conductivity or in the recharge rate. 


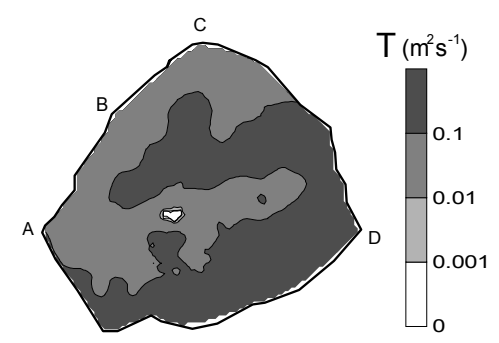

Figure 15: Steady state transmissivity map of the Lez model. The zero transmissivity at the centre of the model is due to a zero aquifer thickness.

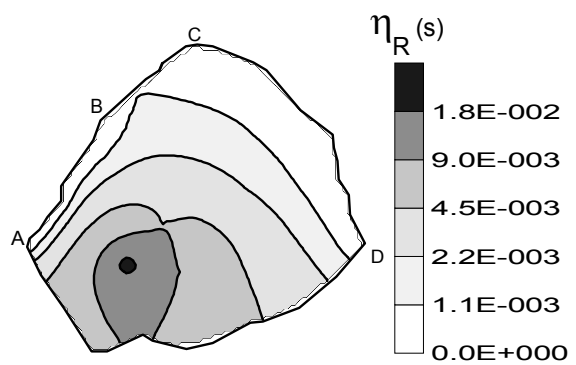

Figure 16: Non-parallel, steady-state mixed confined-unconfined flow. Case of a perturbation in the recharge rate : sensitivity of the hydraulic head. The simulation parameters are given in Table 5 .
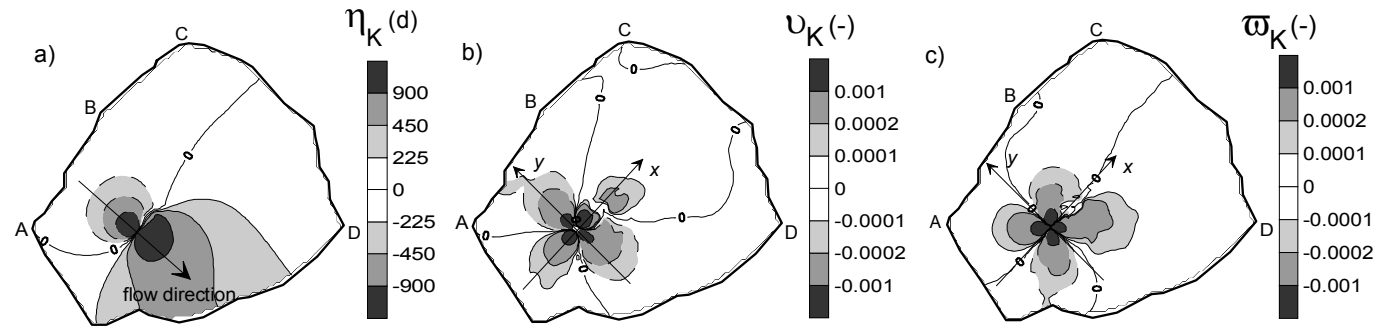

Figure 17: Non-parallel, steady-state mixed confined-unconfined flow. Case of a perturbation in the hydraulic conductivity : a) sensitivity of the hydraulic head, b) sensitivity of the longitudinal flow velocity, c) sensitivity of the transverse flow velocity. Dashed and solid lines indicate negative and positive values respectively. The simulation parameters are given in Table 5 .

\begin{tabular}{lll}
\hline Symbol & Meaning & Value \\
\hline$K$ & Hydraulic conductivity & $10^{-4} \mathrm{~m} / \mathrm{s}$ \\
$R$ & Recharge rate & $9.310^{-9} \mathrm{~m} / \mathrm{s}$ \\
$Q$ & Lez spring pumping rate & $2.2 \mathrm{~m}^{3} / \mathrm{s}$ \\
$k_{O}$ & Perturbation in the hydraulic conductivity & $-2.510^{-5} \mathrm{~m} / \mathrm{s}$ \\
$r_{o}$ & Perturbation in the recharge rate & $2.310^{-10} \mathrm{~m} / \mathrm{s}$ \\
$\nabla H$ & Hydraulic head gradient at the centre of the perturbed area & $2.5 \mathrm{~mm} / \mathrm{m}$ \\
$H_{A}$ & Prescribed hydraulic head at point A & $70 \mathrm{~m}$ \\
$H_{B}$ & Prescribed hydraulic head at point B & $126 \mathrm{~m}$ \\
$H_{C}$ & Prescribed hydraulic head at point C & $115 \mathrm{~m}$ \\
$H_{D}$ & Prescribed hydraulic head at point D & $15 \mathrm{~m}$ \\
\hline
\end{tabular}

Table 5: Non-parallel, steady-state mixed confined-unconfined flow. Parameters for the application example described in Section 6.3: case of a perturbation in the hydraulic conductivity or in the recharge rate. The hydraulic head prescribed along the borders $(A B)$ and $(\mathrm{CD})$ is interpolated linearly between $H_{A}$ and $H_{B}$ along the border $(A B)$, and between $H_{C}$ and $H_{D}$ along the border (CD). (BC) and (AD) are no-flux boundaries. 


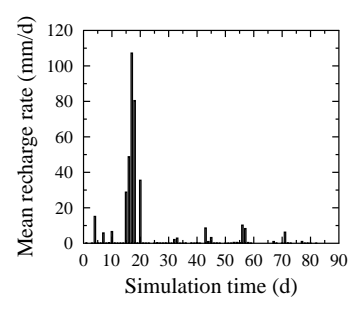

Figure 18: Non-parallel, transient mixed confined-unconfined flow. Case of a perturbation in the hydraulic conductivity. Mean daily recharge rate over the Lez aquifer during the simulation period.

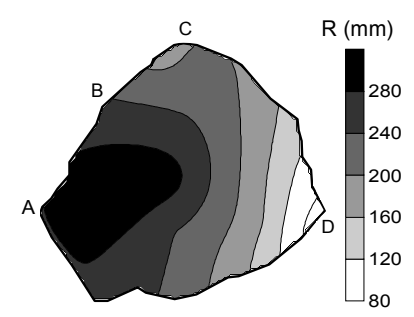

Figure 19: Non-parallel, transient mixed confined-unconfined flow. Case of a perturbation in the hydraulic conductivity. Cumulated rainfall over the Lez aquifer from day 15 to day 17 .

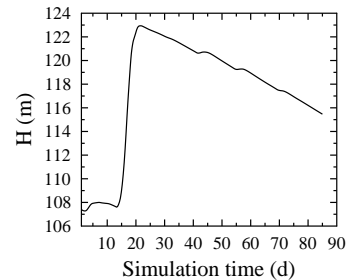

Figure 20: Non-parallel, transient mixed confined-unconfined flow. Case of a perturbation in the hydraulic conductivity. Hydraulic head at the centre of perturbed area, from day 1 to day 85 (application example $6.4)$.

rainfall distribution in Figure 19). Figure 20 shows the variations of the hydraulic head at the centre of the perturbed area during the simulation. Figure 21 shows the hydraulic head sensitivity pattern generated by a perturbation in the hydraulic conductivity at day 21 and day 60. The experimental transient sensitivity pattern matches the theoretical patterns established in Section 4.1 for uniform properties and steady-state flow.
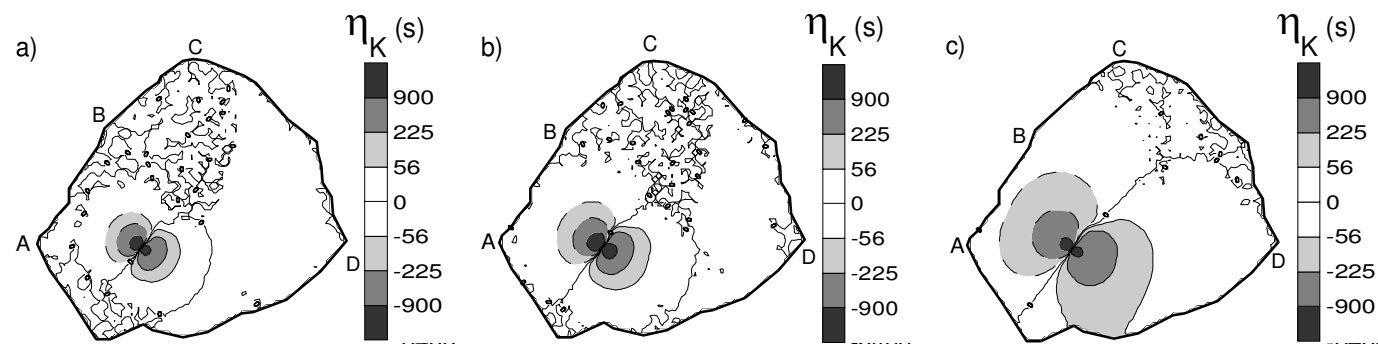

Figure 21: Non-parallel, transient mixed confined-unconfined flow. Case of a perturbation in the hydraulic conductivity. Sensitivity of the hydraulic head : a) at day 10, b) at day 21, c) at day 80 . The simulation parameters are given in Table 6

\subsection{Sensitivity to boundary conditions}

The hydraulic head sensitivity patterns generated by a perturbation in the boundary conditions are investigated over a schematic representation of the Lez karst aquifer system. Experimental sensitivities are computed using the same methodology as in Section 6.1. The parameters are given in Table 7 .

Figure 22a shows the hydraulic head sensitivity pattern generated by a perturbation $H_{o}$ in the hydraulic head prescribed along the western boundary. The perturbation yields a unit sensitivity value along the boundary, as seen in Section 5.1, while the hydraulic head prescribed along the eastern boundary yields a sensitivity value of zero along this boundary. The decrease of the sensitivity inside the model is quasi-linear, which is in agreement whith the results of Section 5.2. Indeed, the geometry of the sensitivity boundary conditions is quasi one-dimensional. Assume now that a flux condition is prescribed along the western boundary. The flux values are chosen so that the hydraulic head pattern remains on the whole the same. The resulting sensitivity pattern is similar to the former one, as the sensitivity decreases linearly from the perturbed boundary to the eastern prescribed 
head boundary (Figure 22b). On the other hand, the sensitivity pattern generated by a perturbation in the flux condition prescribed along the northern border differs from the previous ones (see Figure 22c). This confirms that the boundary condition sensitivity pattern only depends on the geometry of the problem, as established in Section 5.1.

The next simulations investigate the effect of the transmissivity heterogeneity in the quasi 1-D geometry. The transmissivity in the area close to the perturbed boundary is $1 \%$ of the transmissivity value in the rest of the model (see Figure 23a). The hydraulic head sensitivity pattern generated by a perturbation in the hydraulic head prescribed along the western border is shown in Figure 23b. Compared to the case of an homogeneous transmissivity field (Figure 22a), the sensitivity to the head boundary condition is strongly attenuated by the low transmissive area, as predicted in Section 5.2. Assume now that the condition prescribed along the western boundary is a prescribed flux condition. Figure 23c shows the hydraulic head sensitivity pattern generated by a perturbation in this flux boundary condition. The comparison with the homogeneous case (Figure 22b) shows that the change in the transmissivity value in the area close to the boundary has no effect on the sensitivity value in the rest of the model, as established in Section 5.2.

The case of a 2-D geometry is investigated in the next simulations. Figure 24a shows the transmissivity distribution. Figures $24 \mathrm{~b}$ and $24 \mathrm{c}$ show the sensitivity patterns generated by a perturbation in the hydraulic head or in the flux prescribed along the northern border. The resulting sensitivity patterns match the 1-D case, which confirms the validity of these results in the $2-\mathrm{D}$ case.
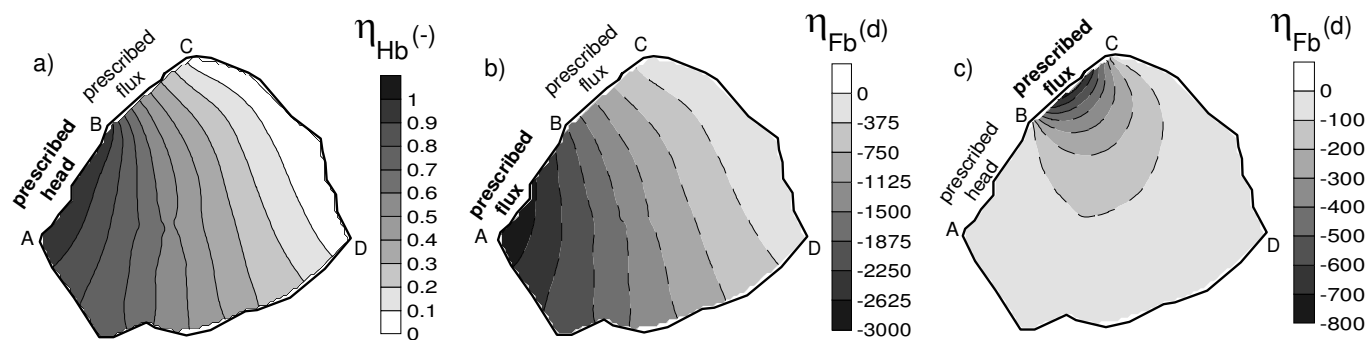

Figure 22: Steady-state, mixed confined-unconfined non-parallel flow. Case of a perturbation in the boundary conditions. Sensitivity of the hydraulic head to a perturbation : a) in the western border prescribed head boundary condition, b) in the western border prescribed flux condition, c) in the northern border prescribed flux condition. A zero flux is prescribed along the boundary (AD). The hydraulic head is fixed along the boundary (CD). The nature of the boundary condition prescribed along (AB) and (BC) depends on the simulation and is indicated on the corresponding figure (bold characters are used for the perturbed condition). Dashed and solid lines indicate negative and positive values respectively.

\begin{tabular}{lll}
\hline Symbol & Meaning & Value \\
\hline$K$ & Hydraulic conductivity & $10^{-4} \mathrm{~m} / \mathrm{s}$ \\
$R$ & Recharge rate & $9.310^{-9} \mathrm{~m} / \mathrm{s}$ \\
$S$ & Specific yield & $1.410^{-2}$ \\
$Q$ & Lez spring pumping rate & $2.2 \mathrm{~m}^{3} / \mathrm{s}$ \\
$k_{O}$ & Perturbation in the hydraulic conductivity & $-2.510^{-5} \mathrm{~m} / \mathrm{s}$ \\
$H_{A}$ & Prescribed hydraulic head at point A & $70 \mathrm{~m}$ \\
$H_{B}$ & Prescribed hydraulic head at point B & $126 \mathrm{~m}$ \\
$H_{C}$ & Prescribed hydraulic head at point C & $115 \mathrm{~m}$ \\
$H_{D}$ & Prescribed hydraulic head at point D & $15 \mathrm{~m}$ \\
\hline
\end{tabular}

Table 6: Non-parallel, transient mixed confined-unconfined flow. Parameters for the application example described in Section 6.4 : case of a perturbation in the hydraulic conductivity. The hydraulic head prescribed along the borders $(A B)$ and $(\mathrm{CD})$ is interpolated linearly between $H_{A}$ and $H_{B}$ along the border $(A B)$, and between $H_{C}$ and $H_{D}$ along the border (CD). (BC) and (AD) are no-flux boundaries. 

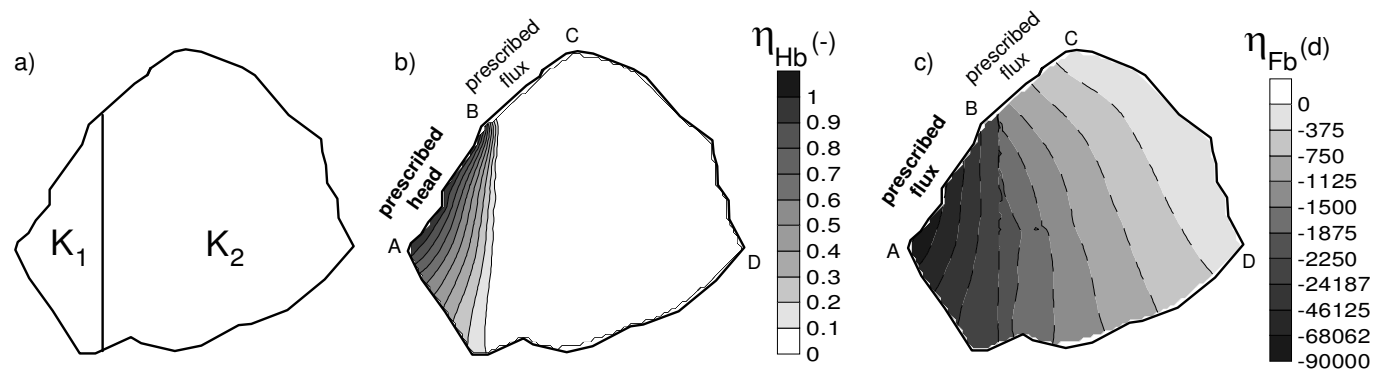

Figure 23: Steady-state, mixed confined-unconfined non-parallel flow. Case of a perturbation in the boundary conditions. Sensitivity of the hydraulic head to a perturbation in the western boundary condition, for a non homogeneous transmissivity distribution : a) definition sketch for the transmissivity distribution $\left(\mathrm{K}_{1} \ll \mathrm{K}_{2}\right)$, b) sensitivity pattern generated by a perturbation in the hydraulic head prescribed along the western boundary, c) sensitivity pattern generated by a perturbation in the flux prescribed along the western boundary. A zero flux is prescribed along the boundary (AD). The hydraulic head is fixed along the boundary (CD). The nature of the boundary condition prescribed along (AB) and (BC) depends on the simulation and is indicated on the corresponding figure (bold characters are used for the perturbed condition). Dashed and solid lines indicate negative and positive values respectively.

a)

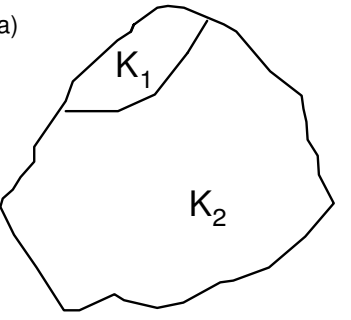

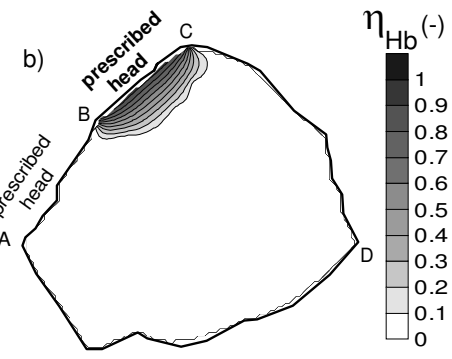

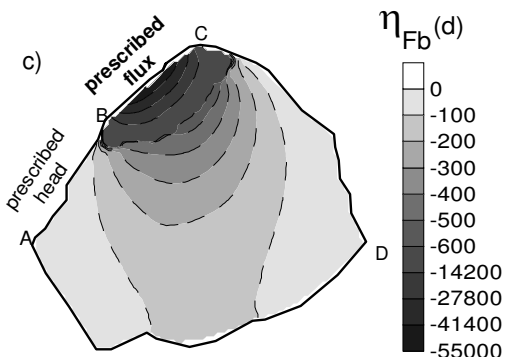

Figure 24: Steady-state, mixed confined-unconfined non-parallel flow. Case of a perturbation in the boundary conditions. Sensitivity of the hydraulic head to a perturbation in the northern boundary condition, for a non homogeneous transmissivity distribution : a) definition sketch for the transmissivity distribution $\left(\mathrm{K}_{1} \ll \mathrm{K}_{2}\right)$, b) sensitivity pattern generated by a perturbation in the hydraulic head prescribed along the northern boundary, c) sensitivity pattern generated by a perturbation in the flux prescribed along the northern boundary. A zero flux is prescribed along the boundary (AD). The hydraulic head is fixed along the boundary (CD). The nature of the boundary condition prescribed along (AB) and (BC) depends on the simulation and is indicated on the corresponding figure (bold characters are used for the perturbed condition). Dashed and solid lines indicate negative and positive values respectively. 


\section{Conclusions}

The theoretical developments presented in Sections 2 to 5 indicate that the shape and extent of the sensitivity pattern depend on the nature of both the perturbed parameter $(K, z, e$ or $R)$ and the variable of interest (hydraulic head or Darcy velocity). Although derived for simple, homogeneous systems and steady-state parallel flow, these results have been validated for complex real-world systems under both non-parallel and transient flow conditions (Section 6), which allows some guidelines to be proposed for the calibration of groundwater flow models and for observation well network design. Items 1 to 5 focus on the sensitivity to the flow parameters. Item 6 deals with the sensitivity to the boundary conditions. Item 7 concludes on the possibility to discriminate between the potential sources of model error.

1. The informations conveyed by hydraulic head and flow velocity measurements are complementary. As an example, consider the case when the model hydraulic conductivity is calibrated against piezometric level measurements. Regions with wrong $K$ estimation located in the transverse and diagonal directions from the measurement point with respect to the flow direction may remain unnoticed, unless tracing experiments are performed.

2. For the case where model results do not coincide with the measurements, the region where the modelled hydraulic conductivity, aquifer thickness or bedrock elevation may be wrongly estimated must be sought downstream and upstream of the measurement point for a discrepancy in the hydraulic head. Besides, the origin of a discrepancy in the longitudinal flow velocity must be sought in both longitudinal and transverse directions to the flow, whereas the origin of a discrepancy in the transverse flow velocity must be sought in diagonal directions to the flow. Furthermore, if the discrepancy stems from bad recharge estimation, the region with wrong recharge estimation may be located anywhere in the model. The possibility to discriminate between the different sources of error is discussed in item 7 .

3. Confined and unconfined aquifers behave differently with respect to sensitivity propagation. While the sensitivity to a perturbation in $K$ (or $z$, or $e$ ) of both the hydraulic head and the flow velocities propagates with the same intensity upstream and downstream of the perturbed area in confined aquifers, it extends further downstream than upstream in unconfined aquifers. Consequently, the area in which the wrongly estimated parameter ought to be sought extends farther downstream than upstream for unconfined aquifers, while it extends equally in both directions in confined aquifers.

4. Model calibration should take into account the existence of dead zones in the sensitivity patterns of $K, z$ and $e$. Indeed, suppose that the model is to be tuned so as to fit the hydraulic head at a given location (target point). Then calibrating $K$ (or $z$, or $e$ ) in the transverse direction to the flow with respect to the target point would be meaningless, as the sensitivity of the hydraulic head to these parameters is zero. Moreover, doing so could lead to assign physically unrealistic values to the parameter in order to make model results fit the measurements.

5. Sampling network design should be adapted to the nature of the problem addressed. For instance, water resource assessment requires an accurate estimation of the hydraulic heads. In this case, the parameters $K, z$, and $e$ have to be estimated upstream and downstream of the target point principally. Besides, the key variable in solute transport problems is the velocity field. Then the parameters $K, z$, and $e$ must be evaluated principally in the longitudinal and transverse directions to the flow. The confined or unconfined character of the aquifer should also be taken into account, as the magnitude of the sensitivity is larger downstream than upstream of the target point for unconfined aquifers. 
6. As far as the sensitivity to the boundary conditions is concerned, no general sensitivity pattern can be established in the two-dimensional case. Indeed, the sensitivity equation cannot be solved without specifying the boundary conditions, i.e. the model geometry. The uncertainty in the simulated hydraulic head stemming from an uncertain head boundary is minimized if the boundary lies in a region with low transmissivity. On the other hand, the uncertainty in the simulated hydraulic head stemming from an uncertain flux boundary is minimized for low average transmissivity between the target point and a known head condition. The consequences for model design are that : (i) whenever possible, uncertain head boundaries should be located in low transmissivity regions, so that the error on the hydraulic heads will not propagate to higher transmissivity regions, (ii) although the influence of uncertain flux boundaries on hydraulic heads cannot be minimized, such boundaries should also be located in low transmissivity regions, if possible. Indeed, the uncertainty on the boundary condition will be more easily reduced if hydraulic head measurements are performed in a low transmissivity, high sensitivity gradient and high sensitivity value region.

7. The fact that the sensitivity pattern depends on the nature of the perturbed parameter may be used to discriminate between wrong hydraulic conductivity (or $z$, or e) estimation, wrong recharge and wrong boundary condition estimation, based on measurements of the flow variable at different locations. Indeed, the propagation of the error along the flow (if the observed variable is the hydraulic head) or in directions transverse to the flow (if the observed variable is the flow velocity) is typical of a wrongly estimated hydraulic conductivity (or $z$, or $e$ ), while an isotropic propagation of the error is typical of wrong recharge estimation. Classical nine-spot well pattern (see spatial setting in Figure 25) could allow for the observation of the error pattern, therefore enabling the identification of the error source. The error pattern stemming from wrong boundary condition estimation depends on the problem geometry, which makes it more difficult to identify. Yet wrong boundary condition estimation may influence a wider area than wrong flow parameter estimation. Nested observation networks could therefore help resolve the ambiguity between wrong boundary condition and wrong parameter estimation. Such networks were primarily designed by the petroleum industry to maximize the oil recovery by water flooding [40,41]. They are also used for the characterisation of the spatial variability of transmissivity fields by means of geostatistical moment analysis. Indeed, performing small-scale pumping tests at various locations across an aquifer and analyzing them by means of geostatistical moment analysis requires the availability of numerous transmissivity data spread more or less evenly across the site of interest [42]. The present work shows that existing nested well networks could offer insights on the nature and location of the aquifer heterogeneities even when used for passive monitoring.

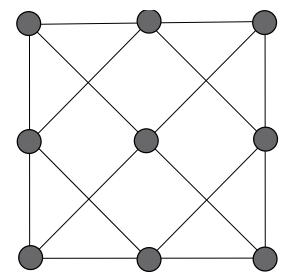

b)

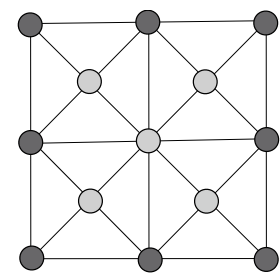

Figure 25: Observation wells network patterns : a) nine-spot pattern, b) five-spot pattern (light dots) and nested five-spot pattern (light and dark dots). 


\section{Acknowledgements}

This work was supported by a $\mathrm{PhD}$ grant from the French Ministère de l'Education Nationale et de la Recherche (allocation couplée number 30368-2008). The authors gratefully thank the four anonymous reviewers for their helpful comments, as well as Frederick Delay (Université de Poitiers) and Patrick Lemonnier (IFP) for their help in finding references on observation wells network patterns.

\section{Appendix A. Sensitivity source term}

Appendix A.1. Source term derivation for a perturbation in the hydraulic conductivity under confined, parallel flow conditions

The source term $q$ generated by a perturbation in the hydraulic conductivity under confined conditions is

$$
q=\nabla[e \varepsilon \nabla H]
$$

Assume that the aquifer thickness is homogeneous over the perturbed area. Also assume that the flow is parallel and directed along the $y$-axis over the perturbed area. Then the hydraulic head gradient is constant and the source term reduces to

$$
q=e \frac{\partial H}{\partial y} \frac{\partial \varepsilon}{\partial y}
$$

The value of the derivative of $\varepsilon$ in the $y$ direction is $+1 / L$ over face 2 , and $-1 / L$ over face 4 , which leads to

$$
q=\left\{\begin{array}{rr}
-\frac{e}{L} \frac{\partial H}{\partial y} & (\text { face } 4) \\
+\frac{e}{L} \frac{\partial H}{\partial y} & \text { (face 2) }
\end{array}\right.
$$

Consequently, the source term integral over each face $Q$ is

$$
Q= \begin{cases}-e L \frac{\partial H}{\partial y} & (\text { face } 4) \\ +e L \frac{\partial H}{\partial y} & (\text { face } 2)\end{cases}
$$

An equivalent source term pattern may be obtained by lumping the source term into source terms located at the gravity centres of the faces. The equivalent source term pattern is made of two point sources of intensity $-e L \partial H / \partial y$ and $+e L \partial H / \partial y$ located at $(0,-2 L / 3)$ and $(0,2 L / 3)$ respectively.

Appendix A.2. Source term derivation for a perturbation in the hydraulic conductivity under confined, radial flow conditions

The second-order terms may be expressed as

$$
\begin{aligned}
\rho_{1} & =-\frac{\varepsilon}{r^{\prime 2}} \\
\rho_{22} & =\frac{1}{r^{\prime}} \frac{\partial \varepsilon}{\partial r^{\prime}}-\frac{1}{r_{o}^{\prime}} \frac{\partial \varepsilon}{\partial r^{\prime}}
\end{aligned}
$$


Over face 4 the source term $\rho_{1}$ may be approximated as

$$
\begin{aligned}
& \rho_{1} \approx-\frac{\varepsilon}{{r_{o}^{\prime}}^{2}} \\
& \rho_{1} \approx-\frac{1}{{r_{o}^{\prime}}^{2}}\left(1-\frac{y}{L}\right)
\end{aligned}
$$

The integral of $\rho_{1}$ over face 4 may be expressed as

$$
\begin{aligned}
& \rho_{1} \approx-\frac{1}{r_{o}^{\prime 2}} \int_{0}^{L}\left(1-\frac{y}{L}\right) 2 y d y \\
& \rho_{1} \approx-\frac{1}{3} \frac{L^{2}}{{r_{o}^{\prime}}^{2}}
\end{aligned}
$$

Over face 4 the source term $\rho_{22}$ may be approximated as

$$
\begin{aligned}
& \rho_{22} \approx-\frac{1}{r_{o}^{\prime} L}\left(1-\frac{y}{r_{o}^{\prime}}\right)+\frac{1}{r_{o}^{\prime} L} \\
& \rho_{22} \approx+\frac{y}{r_{o}^{\prime 2} L}
\end{aligned}
$$

The integral of $\rho_{22}$ over face 4 may be expressed as

$$
\begin{aligned}
& \rho_{22} \approx+\frac{1}{r_{o}^{\prime 2} L} \int_{0}^{L} 2 y^{2} d y \\
& \rho_{22} \approx+\frac{2}{3} \frac{L^{2}}{{r_{o}^{\prime}}^{2}}
\end{aligned}
$$

Similarly, the integrals of $\rho_{1}$ and $\rho_{22}$ over face 2 may be may be expressed as

$$
\begin{array}{r}
\rho_{1} \approx-\frac{1}{3} \frac{L^{2}}{{r_{o}^{\prime}}^{2}} \\
\rho_{22} \approx+\frac{2}{3} \frac{L^{2}}{{r_{o}^{\prime}}^{2}}
\end{array}
$$

The overall effect of the second-order source terms is to increase the algebraic value of the first-order sensitivity source terms. This leads to an increase of the absolute value of the sensitivity in the direction of the converging flow path lines, and a decrease in the direction of the diverging flow path lines.

Appendix A.3. Source term derivation for a perturbation in the hydraulic conductivity under unconfined conditions

The source term $q$ generated by a perturbation in the hydraulic conductivity under unconfined conditions is

$$
q=\nabla[(H-z) \varepsilon \nabla H]
$$

Assume that the flow is parallel and directed along the $y$-axis over the perturbed area. Then $(H-z) \nabla H$ is a constant and the source term is equal to zero over the faces 1 and 3 . Over the faces 2 and 4 , the source term may be written as :

$$
q=(H-z)_{o}\left(\frac{\partial H}{\partial y}\right)_{o} \frac{\partial \varepsilon}{\partial y}
$$


where $(H-z)_{o}$ is the aquifer thickness at the centre of the perturbed area and $(\partial H / \partial y)_{o}$ is the value of the hydraulic head gradient at the centre of the perturbed area. The value of the derivative of $\varepsilon$ in the $y$ direction is $+1 / L$ over face 2 , and $-1 / L$ over face 4 , which leads to

$$
q= \begin{cases}-\frac{(H-z)_{o}}{L}\left(\frac{\partial H}{\partial y}\right)_{o} & (\text { face } 4) \\ +\frac{(H-z)_{o}}{L}\left(\frac{\partial H}{\partial y}\right)_{o} & (\text { face } 2)\end{cases}
$$

Consequently, the source term integral over each face $Q$ is

$$
Q= \begin{cases}-L(H-z)_{o}\left(\frac{\partial H}{\partial y}\right)_{o} & (\text { face } 4) \\ +L(H-z)_{o}\left(\frac{\partial H}{\partial y}\right)_{o} & (\text { face } 2)\end{cases}
$$

The equivalent source term pattern is made of two point sources of intensity $-L(H-$ $z)_{o}(\partial H / \partial y)_{o}$ and $-L(H-z)_{o}(\partial H / \partial y)_{o}$ located at $(0,-2 L / 3)$ and $(0,2 L / 3)$ respectively.

Appendix A.4. Sensitivity derivation for a perturbation in the recharge under unconfined, parallel flow conditions

In this paragraph we convert the sensitivity of the flow velocities in the Cartesian coordinate system. The Cartesian coordinates of the unitary radial and tangential vectors are

$$
\begin{aligned}
& e_{\rho}=\left[\begin{array}{c}
-\sin \theta \\
\cos \theta
\end{array}\right] \\
& e_{\theta}=\left[\begin{array}{c}
-\cos \theta \\
-\sin \theta
\end{array}\right]
\end{aligned}
$$

The sensitivities of the radial and tangential flow velocities may thus be expressed in the Cartesian coordinate system as

$$
\begin{aligned}
& \rho_{R}=\frac{2 L^{2}}{3 \pi(H-z)}\left(\frac{1}{r}+\ln \left(\frac{r}{d}\right) \frac{\partial(H-z)}{\partial y} \frac{\cos \theta}{H-z}\right)\left[\begin{array}{c}
-\sin \theta \\
\cos \theta
\end{array}\right] \\
& \theta_{R}=-\frac{2 L^{2}}{3 \pi} \ln \left(\frac{r}{d}\right) \frac{\partial(H-z)}{\partial y} \frac{\sin \theta}{(H-z)^{2}}\left[\begin{array}{c}
-\cos \theta \\
-\sin \theta
\end{array}\right]
\end{aligned}
$$

As $\sin \theta=-x / r$ and $\cos \theta=y / r$, equations (A.16a) and (A.16b) lead to

$$
\begin{aligned}
\nu_{R} & =\frac{2 L^{2}}{3 \pi} \frac{y}{(H-z) r^{2}}+\frac{2 L^{2}}{3 \pi(H-z)^{2}} \frac{\partial(H-z)}{\partial y} \ln \left(\frac{r}{d}\right) \\
\varpi_{R} & =\frac{2 L^{2}}{3 \pi} \frac{x}{(H-z) r^{2}}
\end{aligned}
$$




\section{Appendix B. Sensitivity to boundary conditions}

The following section investigates the effect of the transmissivity heterogeneity over the sensitivity of the hydraulic head to boundary flux conditions and to head-flux relationships, for a one-dimensional problem. The origin of the $n$-axis is taken at the crossing with the perturbed boundary.

Consider the case of a boundary with prescribed flux condition. Assume confined conditions. As the problem is one-dimensional, a head condition must be prescribed at the other edge of the model in order to get a well-posed problem. Denote by $\Gamma_{F}$ the flux boundary, and by $\Gamma_{H}$ the prescribed head boundary. The sensitivity value at $\Gamma_{F}$ is not fixed by the sensitivity boundary condition, while the sensitivity value along $\Gamma_{H}$ is equal to zero (see Section 5.1). Assume that the model transmissivity is not uniform. Denote by $T_{(0)}$ the transmissivity value along $\Gamma_{F}$, and by $T_{(n)}$ the transmissivity value inside the model at the abscissa $n$. Under confined conditions, equation (44a) gives

$$
T_{(0)}(\nabla \eta)_{(0)}=-1
$$

According to equation (46) :

$$
T_{(n)}(\nabla \eta)_{(n)}=T_{(0)}(\nabla \eta)_{(0)} \quad \forall n
$$

which leads to

$$
T_{(n)}(\nabla \eta)_{(n)}=-1
$$

The value of the sensitivity gradient at a given abscissa $n$ inside the model only depends on the transmissivity value at the abscissa $n$. The sensitivity value at the perturbed boundary is not fixed by the boundary conditions. The value of the sensitivity inside the model thus only depends on the average transmissivity value between the unperturbed head boundary and the location at which the sensitivity is investigated.

Consider the case of a boundary with prescribed flux condition, under unconfined conditions. Equation (B.2) is still valid, yet the unconfined conditions yields a new sensitivity boundary condition

$$
T_{(0)}(\nabla \eta)_{(0)}+K_{(0)}(\nabla H)_{(0)} \eta_{(0)}=-1
$$

The sensitivity value at the perturbed boundary can be expressed as

$$
\eta_{(0)}=-\int_{\Gamma_{F}}^{\Gamma_{H}}(\nabla \eta)_{(n)} d n
$$

Combining equations (B.5) and (B.2) leads to

$$
\begin{aligned}
\eta_{(0)} & =-\int_{\Gamma_{F}}^{\Gamma_{H}} \frac{T_{(0)}}{T_{(n)}}(\nabla \eta)_{(0)} d n \\
& =-T_{(0)}(\nabla \eta)_{(0)} \int_{\Gamma_{F}}^{\Gamma_{H}} \frac{1}{T_{(n)}} d n
\end{aligned}
$$

Combining equations (B.4) and (B.6b) leads to

$$
\eta_{(0)}=\int_{\Gamma_{F}}^{\Gamma_{H}} \frac{1}{T_{(n)}} d n\left(1-K_{(0)} \nabla H_{(0)} \int_{\Gamma_{F}}^{\Gamma_{H}} \frac{1}{T_{(n)}} d n\right)^{-1}
$$

The sensitivity value at the perturbed boundary thus depends on the average transmissivity, as in the confined case. The sensitivity value inside the model thus depends on the average 
transmissivity between the unperturbed head boundary and the location at which the sensitivity is investigated.

The case of a head-flux relationship under confined or unconfined conditions can be handled following the same reasoning as above, and yields the same conclusion.

[1] C. D. McElwee, Sensitivity of groundwater models with respect to variations in transmissivity and storage, Water Resour Res 14 (3) (1978) 451-459.

[2] J. Helton, R. Cooke, M. Mac Kay, A. Saltelli, Guest editorial - Sensitivity analysis of model output: SAMO 2004, Reliab Eng Syst Safe 91 (10-11) (2006) 1105-1108. doi:10.1016/j.ress.2005.11.013.

[3] M. Huysmans, T. Madarasz, A. Dassargues, Risk assessment of groundwater pollution using sensitivity analysis and a worst-case scenario analysis, Environ Geol 50 (2) (2006) 180-193. doi:10.1007/ s00254-006-0197-1.

[4] M. D. Gunzburger, Sensitivities, adjoints and flow optimization, Int J Numer Meth Fl 31 (1) (1999) 53-78. doi:10.1002/(SICI) 1097-0363(19990915)31:1<53: :A ID-FLD955>3.0.C0;2-Z.

[5] B. S. Ramarao, A. M. Lavenue, G. De Marsily, M. G. Marietta, Pilot point methodology for automated calibration of an ensemble of conditionally simulated transmissivity fields 1 . Theory and computational experiments, Water Resour Res 31 (3) (1995) 475-493. doi:10.1029/94WR02258.

[6] C. McKeown, R. S. Haszeldine, G. D. Couples, Mathematical modelling of groundwater flow at Sellafield, UK, Eng Geol 52 (3-4) (1999) 231-250. doi:10.1016/S0013-7952(99)00008-3.

[7] P. D. Meyer, A. J. Valocchi, J. Wayland Eheart, Monitoring network design to provide initial detection of groundwater contamination, Water Resour Res 30 (9) (1994) 2647-2659. doi:10.1029/94WR00872.

[8] A. Graettinger, J. Lee, H. Reeves, D. Dethan, Quantitative methods to direct exploration based on hydrogeologic information, J Hydroinformatics 8 (2) (2006) 77-90.

[9] V. Guinot, B. Cappelaere, Sensitivity analysis of 2D steady-state shallow water flow. Application to free surface flow model calibration, Adv Water Resour 32 (4) (2009) 540-560. doi:10.1016/j . advwatres . 2009.01 .005$.

[10] S. Vela, R. McKinley, How areal heterogeneities affect pulse-test results, Soc Petrol Eng J 10 (2) (1970) $181-191$.

[11] M. Yukler, Analysis of error in groundwater modelling, Ph.D. thesis, University of Kansas, Lawrence (1976).

[12] D. S. Oliver, The influence of nonuniform transmissivity and storativity on drawdown, Water Resour Res 29 (1) (1993) 169-178. doi:10.1029/92WR02061.

[13] L. G. Tumlinson, J. L. Osiensky, J. P. Fairley, Numerical evaluation of pumping well transmissivity estimates in laterally heterogeneous formations, Hydrogeol J 14 (1-2) (2006) 21-30. doi:10.1007/ s10040-004-0386-5.

[14] M. Willmann, J. Carrera, X. Sanchez-Vila, E. Vazquez-Suné, On the meaning of the transmissivity values obtained from recovery tests, Hydrogeol J 15 (5) (2007) 833-842. doi:10.1007/ s10040-006-0147-8.

[15] N. K. Copty, P. Trinchero, X. Sanchez-Vila, M. S. Sarioglu, A. N. Findikakis, Influence of heterogeneity on the interpretation of pumping test data in leaky aquifers, Water Resour Res 44 (11) (2008) W11419. doi:10.1029/2008WR007120.

[16] J. J. Butler, W. Z. Liu, Pumping tests in non-uniform aquifers: The linear strip case, J Hydrol 128 (1-4) (1991) 69-99. doi:10.1016/0022-1694(91)90132-2.

[17] J. J. Butler, W. Z. Liu, Pumping tests in nonuniform aquifers: The radially asymmetric case, Water Resour Res 29 (2) (1993) 259-269. doi:10.1029/92WR02128.

[18] J. Sykes, J. Wilson, R. Andrews, Sensitivity analysis for steady state groundwater flow using adjoint operators, Water Resour Res 21 (3) (1985) 359-371.

[19] F. Delay, A. Kaczmaryk, P. Ackerer, Inversion of interference hydraulic pumping tests in both homogeneous and fractal dual media, Adv Water Resour 30 (3) (2007) 314-334. doi:10.1016/j. advwatres.2006.06.008.

[20] J. Jiao, C. Zheng, The different characteristics of aquifer parameters and their implications on pumpingtest analysis, Ground Water 35 (1) (1997) 25-29. doi:0.1111/j.1745-6584.1997.tb00056.x.

[21] D. Khan, C. Deutsch, C. Mendoza, B. Rostron, Approximate sensitivity coefficients for integrating hydraulic head data into geological models, J Hydrol 347 (3-4) (2007) 460-473. doi:10.1016/j. jhydrol.2007.09.043.

[22] C. Leven, Effects of heterogeneous parameter distributions on hydraulic tests - analysis and assessment, Ph.D. thesis, Eberhard Kärls Universität, Tübingen (2002). URL http://w210.ub.uni-tuebingen.de/dbt/volltexte/2003/710/index.html

[23] C. Leven, P. Dietrich, What information can we get from pumping tests ? - Comparing pumping test configurations using sensitivity coefficients, J Hydrol 319 (1-4) (2006) 199-215. doi:10.1016/j. jhydrol.2005.06.030.

[24] J. Knight, G. Kluitenberg, Some analytical solutions for sensitivity of well tests to variations in storativity and transmissivity, Adv Water Resour 28 (10) (2005) 1057-1075. doi:10.1016/j. advwatres.2004.08.018. 
[25] M. Jyrkama, J. Sykes, Sensitivity and uncertainty analysis of the recharge boundary condition, Water Resour Res 42 (W01404) (2006) 1-11. doi:10.1029/2005WR004408.

[26] R. M. Neupauer, J. L. Wilson, Backward probabilistic model of groundwater contamination in nonuniform and transient flow, Adv Water Resour 25 (7) (2002) 733-746. doi:10.1016/S0309-1708(02) $00073-8$.

[27] R. M. Neupauer, J. L. Wilson, Backward location and travel time probabilities for a decaying contaminant in an aquifer, J Contam Hydrol 66 (1-2) (2003) 39-58. doi:10.1016/S0169-7722(03) $00024-X$.

[28] J. Bear, Dynamics of fluids in porous media., American Elsevier, New York, 1972.

[29] N.-Z. Sun, W. W.-G. Yeh, Identification of parameter structure in groundwater inverse problem, Water Resour Res 21 (6) (1985) 869-883. doi:10.1029/WR021i006p00869.

[30] N. Sun, Inverse Problems in Groundwater Modelling, Kluwer Academic Publishers, the Netherlands, 1994

[31] W. W.-G. Yeh, Review of parameter identification procedures in groundwater hydrology : The inverse problem, Water Resour Res 22 (2) (1986) 95-108. doi:10.1029/WR022i002p00095.

[32] P. Garabedian, Partial Differential Equations, John Wiley \& Sons, 1964.

[33] H. Diersch, Feflow : user's manual, Institute for Water Resources Planning and Systems Research, WASY, Berlin (1998)

[34] H. Diersch, Feflow : reference manual, Insitute for Water Resources Planning and Systems Research, WASY, Berlin (1998)

[35] J. Avias, Karstic aquifers of mediterranean type, geological controls : "Lez spring" (NorthMontpellieran karsts, France) example, in: W. Back, J. S. Herman, H. Paloc (Eds.), Hydrogeology of selected karst regions, Vol. 13 of International Contributions to Hydrogeology, International Association of Hydrogeologists, Hannover, 1992, pp. 89-113.

[36] C. Drogue, Contribution à l'étude quantitative des systèmes hydrologiques karstiques, d'après l'exemple de quelques karsts méditerranéens., Ph.D. thesis, Université de Montpellier II, 482p. (1969).

[37] G. Berger, B. Alabouvette, J. Guérangé-Lozes, M. Demange, P. Ambert, Carte géologique de la France (1/250 000), feuille Montpellier (38), Orléans : BRGM, notice explicative par B. Alabouvette, M. Demange, J.Guérangé-Lozes, P.Ambert (2003), 164p. (2001).

[38] M. Séranne, A. Benedicto, C. Truffert, G. Pascal, P. Labaume, Structural style and evolution of the gulf of lion oligo-miocene rifting : Role of the pyrenean orogeny, Mar Petrol Geol 12 (1995) 809-820. doi:10.1016/0264-8172(95)98849-Z

[39] A. Benedicto, M. Séguret, P. Labaume, Tertiary extension within the Alpine Orogen, The Geological Society Special Publication, 1999, Ch. Interaction between faulting, drainage and sedimentation in extensional hanging-wall syncline basins: example of the Oligocene Matelles Basin, Gulf of Lion margin (S.E. France)., pp. 81-108.

[40] M. Muskat, R. Wuckoff, P. Pittsburgh, A theoretical analysis of water-flooding networks, A.I.M.E. Technical publication 507 (1933) 62-77.

[41] M. Muskat, Physical properties of Oil production, McGraw. Hill Book Company, 1949.

[42] M. Riva, A. Guadagnini, J. Bodin, F. Delay, Characterization of the Hydrogeological Experimental Site of Poitiers (France) by stochastic well testing analysis, J Hydrol 369 (1-2) (2009) 154-164. doi: $10.1016 / j \cdot$ jhydrol.2009.02.040. 


\begin{tabular}{lll}
\hline Symbol & Meaning & Value \\
\hline$K$ & Hydraulic conductivity (homogeneous case) & $10^{-4} \mathrm{~m} / \mathrm{s}$ \\
$K_{1}$ & Hydraulic conductivity (heterogeneous case) & $10^{-4} \mathrm{~m} / \mathrm{s}$ \\
$K_{2}$ & Hydraulic conductivity (heterogeneous case) & $10^{-6} \mathrm{~m} / \mathrm{s}$ \\
$R$ & Recharge rate & $9.310^{-9} \mathrm{~m} / \mathrm{s}$ \\
$Q$ & Lez spring pumping rate & $2.2 \mathrm{~m}^{3} / \mathrm{s}$ \\
$H_{O}$ & Perturbation in the hydraulic head & $4 \mathrm{~m}$ \\
$F_{O}$ & Perturbation in the flux & $2.310^{-8} \mathrm{~m} / \mathrm{s}$ \\
$H_{A}$ & Hydraulic head prescribed at point A & $70 \mathrm{~m}$ \\
$H_{B}$ & Hydraulic head prescribed at point B & $126 \mathrm{~m}$ \\
$H_{C}$ & Hydraulic head prescribed at point C & $115 \mathrm{~m}$ \\
$H_{D}$ & Hydraulic head prescribed at point D & $15 \mathrm{~m}$ \\
$F_{A}$ & Flux prescribed at point A & $1.210^{-7} \mathrm{~m} / \mathrm{s}$ \\
$F_{B}$ & Flux prescribed at point B & $2.710^{-7} \mathrm{~m} / \mathrm{s}$ \\
$F_{B C}$ & Flux prescribed along the (BC) border & $0 \mathrm{~m} / \mathrm{s}$ \\
\hline
\end{tabular}

Table 7: Steady-state, mixed confined-unconfined non-parallel flow. Parameters for the application examples described in Section $6.5:$ case of a perturbation in the boundary conditions. The hydraulic head prescribed along the border (CD) is interpolated linearly between $H_{C}$ and $H_{D}$. A zero flux is prescribed along the (AD) border. Depending on the simulation, either the hydraulic head or the flux may prescribed along the borders $(\mathrm{AB})$ and $(\mathrm{BC})$. In any case, the hydraulic head $H$ or the flux $F$ prescribed along the borders $(\mathrm{AB})$ and $(\mathrm{BC})$ are interpolated linearly between their extremities. 